\title{
Optimized finite-difference (DRP) schemes perform poorly for decaying or growing oscillations ${ }^{\text {th }}$
}

\author{
E.J. Brambley ${ }^{\mathrm{a}, *}$ \\ ${ }^{a}$ Department of Applied Mathematics and Theoretical Physics, University of Cambridge, \\ Wilberforce Road, Cambridge, CB3 0WA, United Kingdom
}

\begin{abstract}
Computational aeroacoustics often use finite difference schemes optimized to require relatively few points per wavelength; such optimized schemes are often called Dispersion Relation Preserving (DRP). Similar techniques are also used outside aeroacoustics. Here the question is posed: what is the equivalent of points per wavelength for growing or decaying waves, and how well are such waves resolved numerically? Such nonconstant-amplitude waves are common in aeroacoustics, such as the exponential decay caused by acoustic linings, the $O(1 / r)$ decay of an expanding spherical wave, and the decay of high-azimuthal-order modes in the radial direction towards the centre of a cylindrical duct. It is shown that optimized spatial derivatives perform poorly for waves that are not of constant amplitude, under performing maximal-order schemes. An equivalent criterion to points per wavelength is proposed for non-constant-amplitude oscillations, reducing to the standard definition for constant-amplitude oscillations and valid even for pure growth or decay with no oscillation. Using this definition, coherent statements about points per wavelength necessary for a given accuracy can be made for maximal-order schemes applied to non-constant-amplitude oscillations. These features are illustrated through a numerical example of a one-dimensional wave propagating through a damping region.
\end{abstract}

Keywords: Optimized finite differences, computational aeroacoustics, DRP schemes, damped waves.

\section{Introduction}

Computational aeroacoustics simulations often use finite difference schemes optimized to need relatively few points per wavelength. Given a series of equidistant points $x_{j}=j \Delta x$ and function evaluations $f_{j}=$ $f\left(x_{j}\right)$, an explicit symmetric finite difference scheme of width $2 N+1$ points and coefficients $d_{q}$ numerically approximates the derivative $f^{\prime}\left(x_{j}\right)$ by

$$
f_{j}^{\prime}=\frac{1}{\Delta x} \sum_{q=1}^{N} d_{q}\left(f_{j+q}-f_{j-q}\right)
$$

For such schemes, a function $f(x)=\operatorname{Re}\left(A \mathrm{e}^{\mathrm{i} \alpha x}\right)$ with derivative $f^{\prime}(x)=\operatorname{Re}\left(\mathrm{i} \alpha A \mathrm{e}^{\mathrm{i} \alpha x}\right)$ gives a numerical derivative

$$
f_{j}^{\prime}=\operatorname{Re}\left(\mathrm{i} \bar{\alpha} A \mathrm{e}^{\mathrm{i} \alpha x_{j}}\right), \quad \quad \quad \text { where } \quad \bar{\alpha} \Delta x=2 \sum_{q=1}^{N} d_{q} \sin (\alpha \Delta x q) \text {. }
$$

\footnotetext{
is A preliminary version of the first part of this paper was presented as part of AIAA Paper 2015-2540 at the 21st AIAA/CEAS Aeroacoustics Conference in Dallas, Texas, USA [1].

*Corresponding author. Tel.: +44 1223 760457. Fax.: +44 1223765900

Email address: E.J.Brambley@damtp.cam.ac.uk (E.J. Brambley)
} 


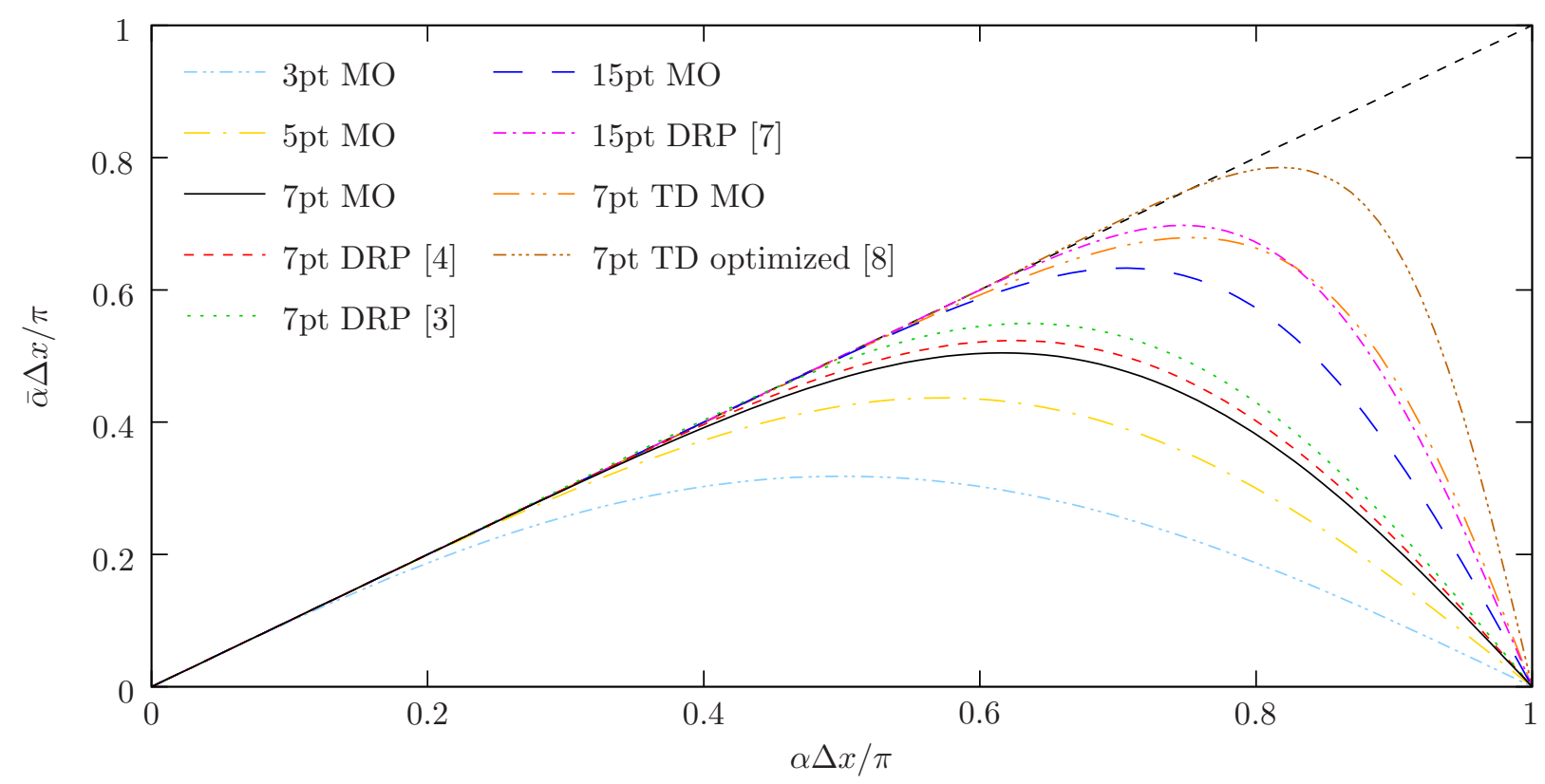

Figure 1: Graph of the numerical modified wavenumber $\bar{\alpha}$ when calculating the derivative of a wave with actual wavenumber $\alpha$ using a finite-difference scheme with points separated by $\Delta x$. The 3, 5, 7 and 15 point MO schemes are standard explicit Maximal-Order schemes, giving 2nd, 4th, 6th and 14th order accuracy respectively; similarly, the 7pt TD MO scheme is a TriDiagonal (implicit) Maximal-Order scheme giving 8th order accuracy. All DRP and optimized schemes plotted in this figure are optimized subject to being 4th order. DRP [3] is optimized for $|\alpha \Delta x / \pi|<0.5$ while DRP [4] is optimized for $|\alpha \Delta x / \pi|<0.35$.

Equation (2) represents the harmonic behaviour of the differentiation scheme, with $\bar{\alpha}$ the numerical modified wavenumber 2]. A classical choice of $d_{q}$ is to give as accurate as possible a derivative in the limit $\Delta x \rightarrow 0$. This choice would give $f_{0}^{\prime}=f^{\prime}(0)+O\left((\Delta x)^{2 N}\right)$, and such $2 N$ th order schemes are referred to here as maximal-order (MO) schemes. An alternative is to only require that $f_{0}^{\prime}=f^{\prime}(0)+O\left((\Delta x)^{2 L}\right)$, and to use the remaining $N-L$ degrees of freedom to optimize the derivative such that $\bar{\alpha}$ is as close to $\alpha$ as possible under some suitable metric. For example, Tam and Webb [3] chose $N=3$ and $L=2$ and optimized the remaining degree of freedom to minimize

$$
\int_{0}^{\eta}(\bar{\alpha} \Delta x-\alpha \Delta x)^{2} \mathrm{~d}(\alpha \Delta x)
$$

with $\eta=\pi / 2$, while Tam and Shen [4] subsequently suggested $\eta=1.1$ gives a more balanced scheme. Minimizing equation (3) is only one possibility, and many other techniques have been employed to optimize $\bar{\alpha}$ in some way [e.g. 5, 6]. These optimized results are often presented as in figure 1

The idea of analysing the harmonic behaviour of numerical derivatives in this way dates back at least to Vichnevetsky and De Schutter [9] and is discussed in detail by Vichnevetsky and Bowles [10], while Holberg [5] considered schemes based on optimizing the numerical group velocity. Lele [1] considered the harmonic accuracy of implicit schemes, which were subsequently optimized by Kim and Lee [8]. Optimized schemes were termed "spectral-like" by Lele [11], "maximum resolution" by Kim and Lee [8], and "extended wavenumber" by Zingg and Lomax [12], while Tam and Webb [3] coined the term Dispersion Relation Preserving (DRP)1. This analysis spurred further investigations, including into 2D spatial derivatives on

\footnotetext{
${ }^{1}$ The term Dispersion Relation Preserving (DRP) has come to be used to describe any optimized finite-difference scheme, although Tam [13] originally intended the term to refer to a joint spatial-temporal scheme, such as discussed by Tam and Webb [3].
} 
triangular grids by Zingg and Lomax 12, into larger width optimized schemes by Bogey and Bailly [14], into non-equidistant grids by Jakobsson [15], and into anisotropic effects on 2D grids with convection by Chenoweth, Soria, and Ooi [16], to name but a few. The field is still being actively investigated, for example by Zhang and Yao [6] using sup-norms and a simulated annealing optimization algorithm, and is regularly rediscovered, as for example by Liu [17] in the geophysics context. Use of optimized finite difference schemes is not limited to aeroacoustics, and examples may be found in Large Eddy Simulation of jets [18, 19] and in fluid convection simulations 20, 21]. For further details of optimized finite difference schemes, see the excellent comparison by Zingg [22], the review by Astley [23], and the recent book by Tam [7].

Optimized DRP schemes are designed to require few points per wavelength for accurate resolution of waves. The present paper is partially motivated by the question: what is the equivalent of points per wavelength for exponentially growing or decaying waves, and how well are such waves resolved numerically? Exponential growth or decay is often found in aeroacoustics. For example, acoustic linings cause an exponential decrease in amplitude as a wave propagates along the lining, and high-order spinning mode in a cylindrical duct (such as commonly excited by an aeroengine rotor) decay exponentially towards the duct centreline. Surface waves over acoustic linings in the direction normal to the lining also exhibit exponential decay 24 26], as do creeping waves giving the diffracted field in the shadow region beyond a smooth obstacle. Weaker decay is often found due to the $O(1 / r)$ decay of a spherical wavefront, which can be rather quick in the vicinity of a point scatterer generating such a spherical wave. As an example of non-constant amplitude waves, Tam et al. 27] considered the effect of liner splices on the decay of an upstream-propagating wave in a cylindrical duct for aeroacoustically-relevant parameters. They showed that liner splices could scatter the incoming wave into less well attenuated waves with an amplitude of $1 \%$ of that of the incident wave, and that these scattered waves could become dominant by the time they reached the end of the liner. In nondimensional terms, the axial wavenumber of the incident wave over the liner that they needed to resolve was $\alpha=-14.7+7.6 \mathrm{i}$ in the no-flow case and $\alpha=-21.0+12.0 \mathrm{i}$ in the case with flow. In both of these cases, the spatial wavenumber clearly has a significant decay rate given by the imaginary part of $\alpha$, and it is unclear how figure 1 applies in such situations; this is the topic of this paper.

\section{Non-constant amplitude harmonic analysis}

Here we consider oscillations that grow or decay as well as oscillating. Such oscillations are characterized by (being the real part of a wave with) a wavenumber $\alpha$ that is complex. While what follows is equally valid for any finite difference scheme on any grid, here for simplicity we consider only symmetric finite difference schemes on regularly spaced grids. We will, however, consider both implicit (i.e. compact) and explicit finite difference schemes, so that our derivatives are given by

$$
f_{j}^{\prime}+\sum_{q=1}^{M} \beta_{q}\left(f_{j+q}^{\prime}+f_{j-q}^{\prime}\right)=\frac{1}{\Delta x} \sum_{q=1}^{N} d_{q}\left(f_{j+q}-f_{j-q}\right) \quad \Rightarrow \quad \bar{\alpha} \Delta x=\frac{2 \sum_{q=1}^{N} d_{q} \sin (\alpha \Delta x q)}{1+2 \sum_{q=1}^{M} \beta_{q} \cos (\alpha \Delta x q)},
$$

where, as before, assuming $f_{j}=\operatorname{Re}\left(A \mathrm{e}^{\mathrm{i} \alpha j \Delta x}\right)$ gives the numerical modified wavenumber $\bar{\alpha}$ with $f_{j}^{\prime}=$ $\operatorname{Re}\left(\mathrm{i} \bar{\alpha} A \mathrm{e}^{\mathrm{i} \alpha j \Delta x}\right)$. A maximal-order scheme of this form has an error of $O\left((\Delta x)^{2 N+2 M}\right)$. For $M=0$ this reduces to the explicit (i.e. non-compact) scheme given in (10) and (2) above, with $f_{j}^{\prime}$ determined explicitly in terms of $f_{j+q}$. The $M=1$ and $M=2$ cases are referred to as tridiagonal and pentadiagonal implicit schemes respectively. Note that equation (4) still holds even for complex $\alpha$, in which case it represents the response of the finite difference scheme to real-valued oscillatory waves with an exponentially decaying or growing amplitude.

\subsection{Performance of optimized and maximal-order schemes for non-constant-amplitude waves}

Considering first the relative phase error $\varepsilon_{p}=|\bar{\alpha} / \alpha-1|$, for several explicit schemes $\varepsilon_{p}$ is plotted in figure 2. As can be seen from figure 2(a-d), the classical maximal-order schemes perform better than the optimized DRP schemes when $\operatorname{Im}(\alpha) \neq 0$ (this is particularly visible along the imaginary $\alpha$ axis). This is not surprising, since the DRP schemes have been optimized to give a good approximation only for real $\alpha$ 
(a) 6th order

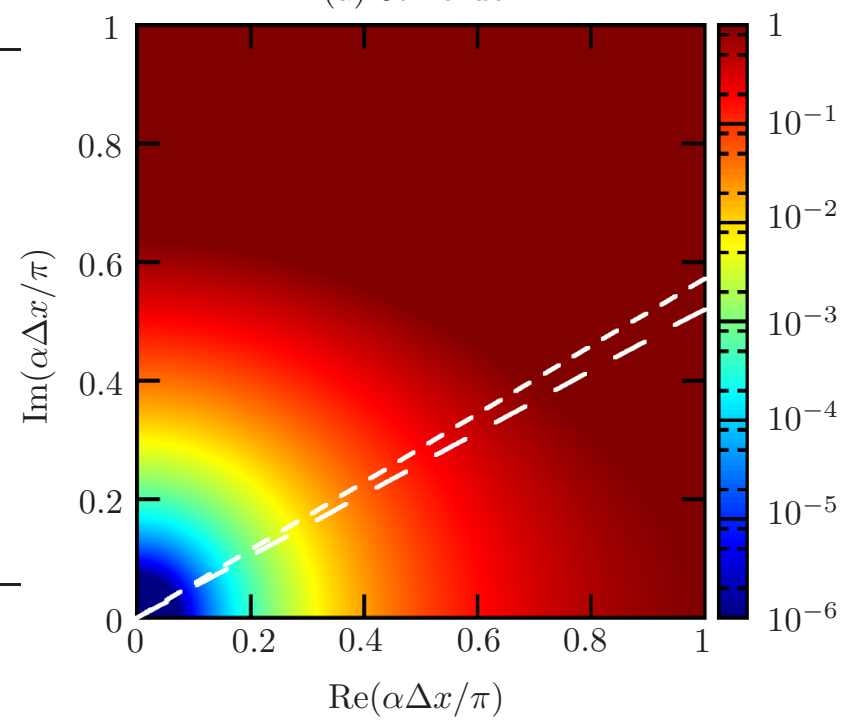

(c) 14 th order

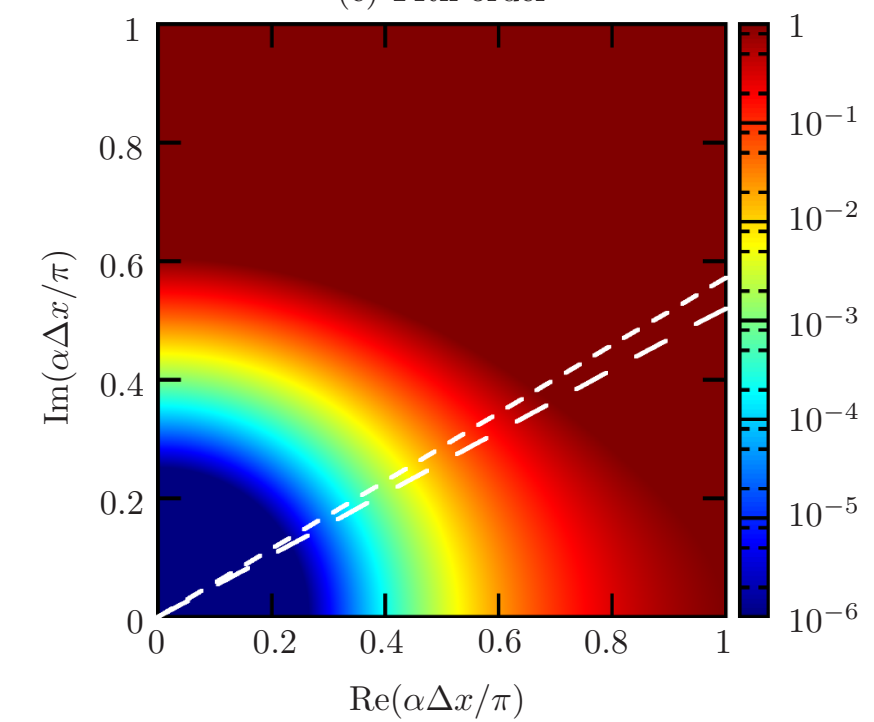

(b) 7-point DRP [4]

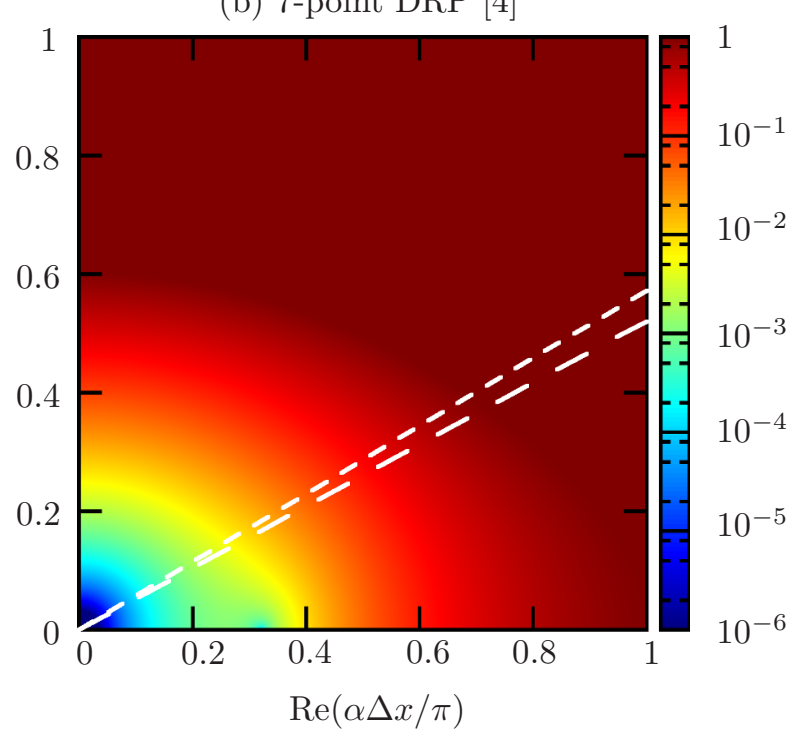

(d) 15-point DRP 7]

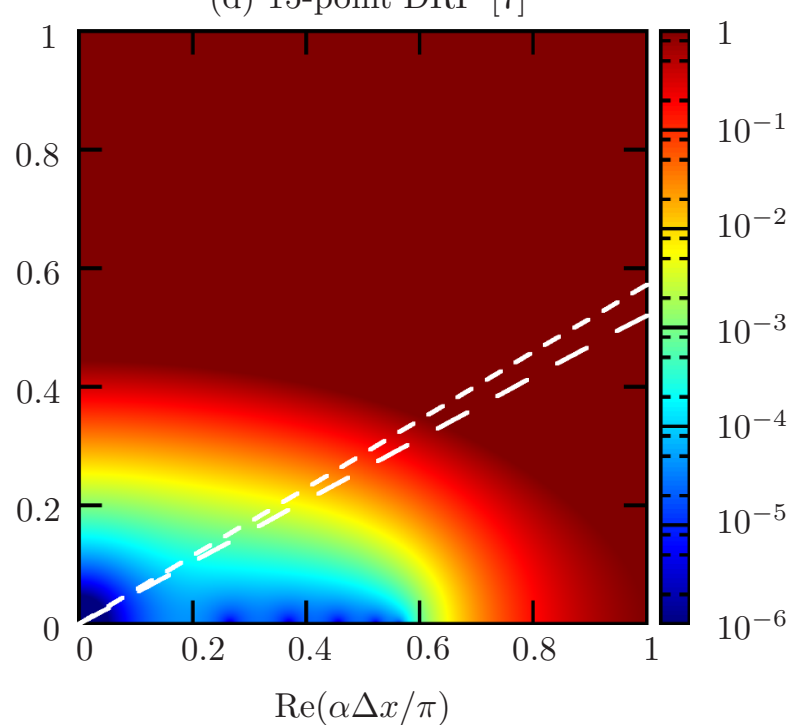

(e) Plots of $(\mathrm{a}-\mathrm{d})$ along the short dashed line

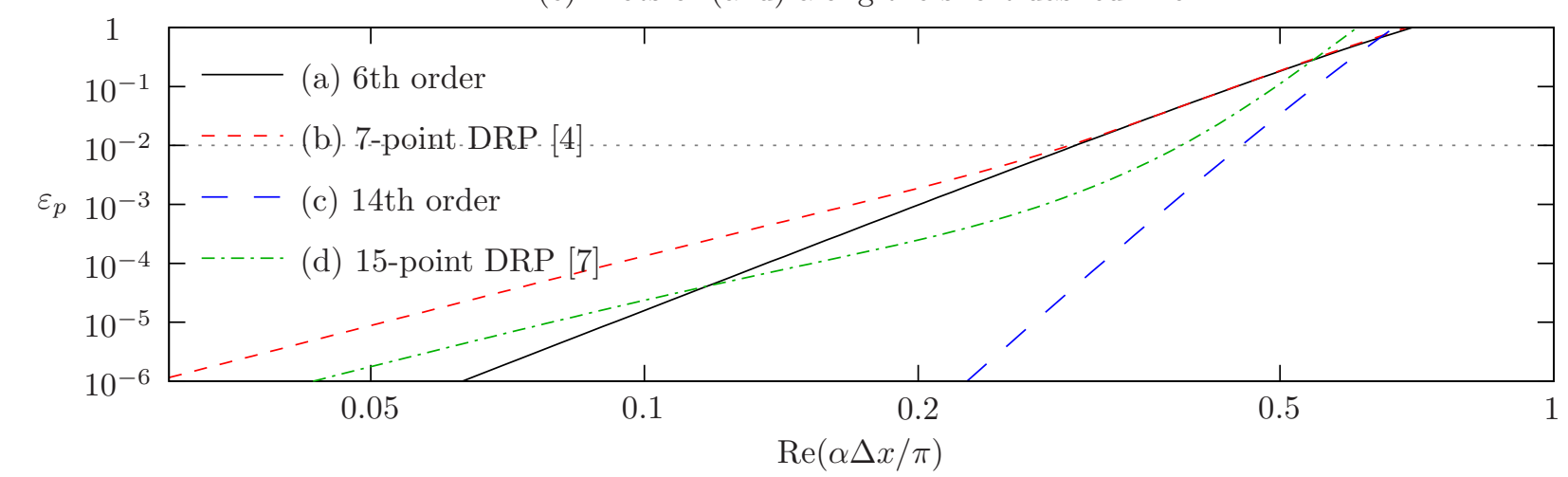

Figure 2: For explicit schemes, plots of $\varepsilon_{p}=|\bar{\alpha} / \alpha-1|$ for complex $\alpha$ on a logarithmic scale, giving the relative accuracy of the numerical derivative. (a) and (b) use 7-point scheme, while (ch and (d) use 15-point schemes. (a) and (c) are maximal-order schemes, while (b) and (d) are optimized 4th order schemes. Short and long dashed lines correspond to $\alpha \Delta x$ for varying $\Delta x$ with and without flow respectively for the axial wavenumbers investigated by Tam et al. [27]. (e) plots the errors of (a-d) along the short dashed line, with the horizontal dotted line indicating a $1 \%$ error. 
and we now see this is at the expense of behaviour for complex $\alpha$. A similar situation is seen in figure 3(a-d) for implicit schemes. In both figures $2(\mathrm{a}-\mathrm{d})$ and $3(\mathrm{a}-\mathrm{d})$ the dashed lines denote the axial wavenumbers of the simulations by Tam et al. [27]. To show further detail, subfigures 2(e) and 3(e) plot the error $\varepsilon_{p}$ along the short dashed lines (corresponding to the with flow case of [27]), together with the relatively lenient $1 \%$ accuracy requirement needed to begin to capture the scattered waves in this case. The classical maximal-order schemes are seen to perform better than the optimized DRP schemes for all step sizes $\Delta x$, and significantly better for smaller values of $\Delta x$.

\subsection{Comparison of optimized and maximal-order schemes for non-constant-amplitude waves}

Possibly a more relevant summary is to consider which of the maximal-order or DRP schemes is more accurate for a given $\alpha$. Using the relative phase error $\varepsilon_{p}$ as the measure of accuracy, this is plotted in figure 4 for explicit schemes, and in figure 5 for implicit schemes. Only values of $\alpha$ for which at least one scheme gives at least $1 \%$ relative accuracy are plotted, in line with the $1 \%$ accuracy requirement from the example of Tam et al. [27]. Figures 4 and 5 only compare schemes that require the same computational effort, and so a gain in accuracy is equivalent to quicker computation at the same accuracy. A more detailed comparison of computational effort is given in section 3.3 .

The region of $\alpha$ for which the optimized DRP schemes are more accurate than the classical maximalorder schemes can be seen from figures 4 and 5 to be rather limited in all cases. Several other schemes have been investigated, including those based on trigonometric interpolation by Tang and Baeder [28], and similar results to those presented here are seen in all cases considered. In all cases, and for any resolution $\Delta x$, the optimized schemes are seen to give worse phase accuracy than the maximal-order schemes for the parameters simulated by Tam et al. [27], which lie along the dashed lines in figure 25 .

The same is true when the relative group velocity error is considered. The relative group velocity is given by differentiating (41),

$$
\frac{\mathrm{d} \bar{\alpha}}{\mathrm{d} \alpha}=\frac{\left(2 \sum_{q=1}^{N} q d_{q} \cos (\alpha \Delta x q)\right)\left(1+2 \sum_{q=1}^{M} \beta_{q} \cos (\alpha \Delta x q)\right)+\left(2 \sum_{q=1}^{N} d_{q} \sin (\alpha \Delta x q)\right)\left(2 \sum_{q=1}^{M} q \beta_{q} \sin (\alpha \Delta x q)\right)}{\left(1+2 \sum_{q=1}^{M} \beta_{q} \cos (\alpha \Delta x q)\right)^{2}} .
$$

We define the relative group velocity error following Trefethen [29] and Holberg [5] as $\varepsilon_{g}=|\mathrm{d} \bar{\alpha} / \mathrm{d} \alpha-1|$. Wave packets, wave fronts and wave energy all travel at the numerical group velocity, and Trefethen [29] concluded group velocity errors gave "a quantitative understanding of differencing errors in wave propagation problems, of the appearance of parasitic waves, of the anisotropic behavior of multidimensional difference schemes, and of instability in initial boundary value problem". Holberg [5] found that constraining the group velocity error was a stricter constraint than constraining the phase velocity error. Here, similar plots to figures 4 and 5 but for the group velocity error $\varepsilon_{g}$ are plotted in figures 6 and 7 The original DRP scheme of Tam and Webb [3] and each of the optimized tri-diagonal implicit schemes can be seen to fare particularly poorly with respect to the group velocity error.

\subsection{A points-per-wavelength equivalent for non-constant-amplitude waves}

Figures 2 and 3 suggest that the accuracy of maximal-order schemes depends on $|\alpha \Delta x|$ but is insensitive to $\arg (\alpha \Delta x)$ at least for small $|\alpha \Delta x|$. Hence, the equivalent of points per wavelength for non-constantamplitude waves is suggested here to be the number of Points Per Complex Wavelength, PPCW $=2 \pi /|\alpha \Delta x|$, which reduces to the usual definition for points per (real) wavelength when $\alpha$ is real. Using this definition, a certain accuracy can be guaranteed for a certain number of points per complex wavelength if a maximal-order scheme is used; for example, for the 7-point maximal order scheme, $2 \pi /|\alpha \Delta x|>6.1$ ensures that $\varepsilon_{p}<0.01$. This definition is however not helpful for optimized DRP schemes, since their accuracy depends both on $|\alpha \Delta x|$ and $\arg (\alpha \Delta x)$; for example, the DRP scheme of Tam and Shen [4] needs $2 \pi /|\alpha \Delta x|>7.2$ to ensure $\varepsilon_{p}<0.01$ for any $\arg (\alpha \Delta x)$, but $2 \pi /(\alpha \Delta x)>4.9$ is sufficient to ensure $\varepsilon_{p}<0.01$ provided $\alpha$ is real. A summary of the required resolution for various spatial derivatives is given in table 1 It is therefore proposed here that, unless specific a priori knowledge of the exact complex wavenumbers present in all directions (e.g. 
(a) 7-point 8th order (MO)

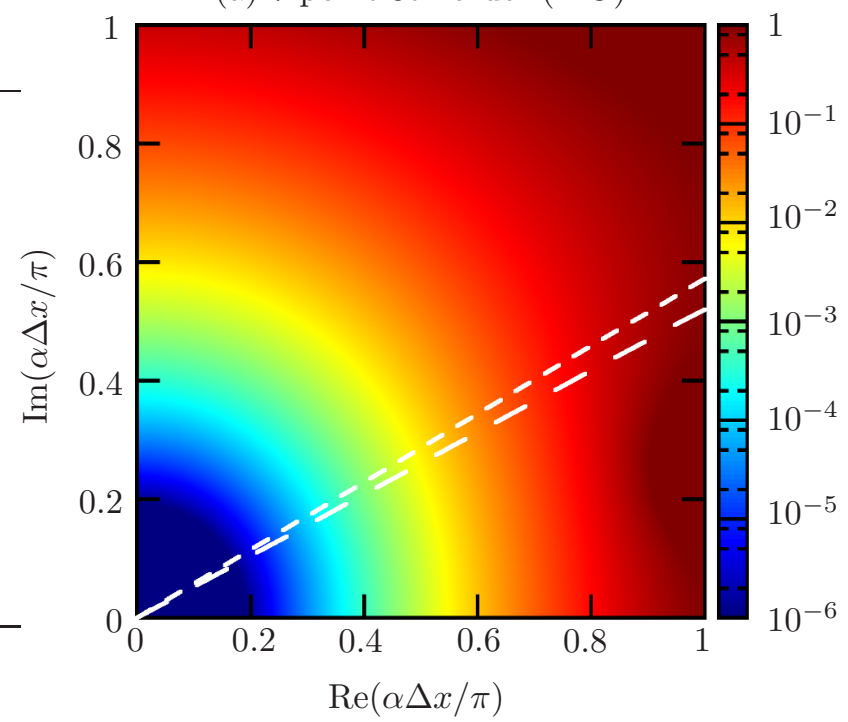

(c) 7-point 2nd order (optimized)

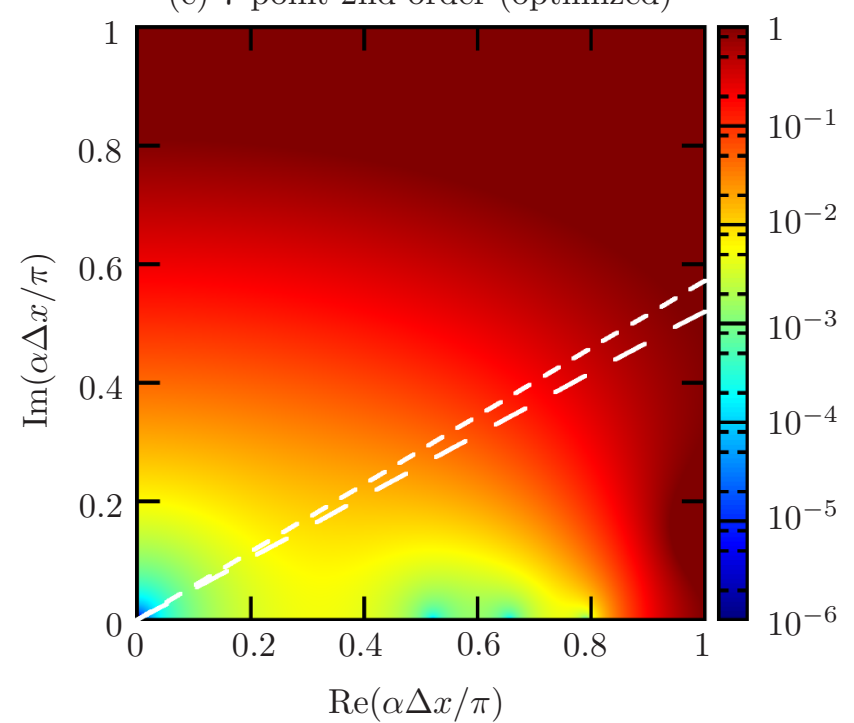

(b) 7-point 6th order (optimized)

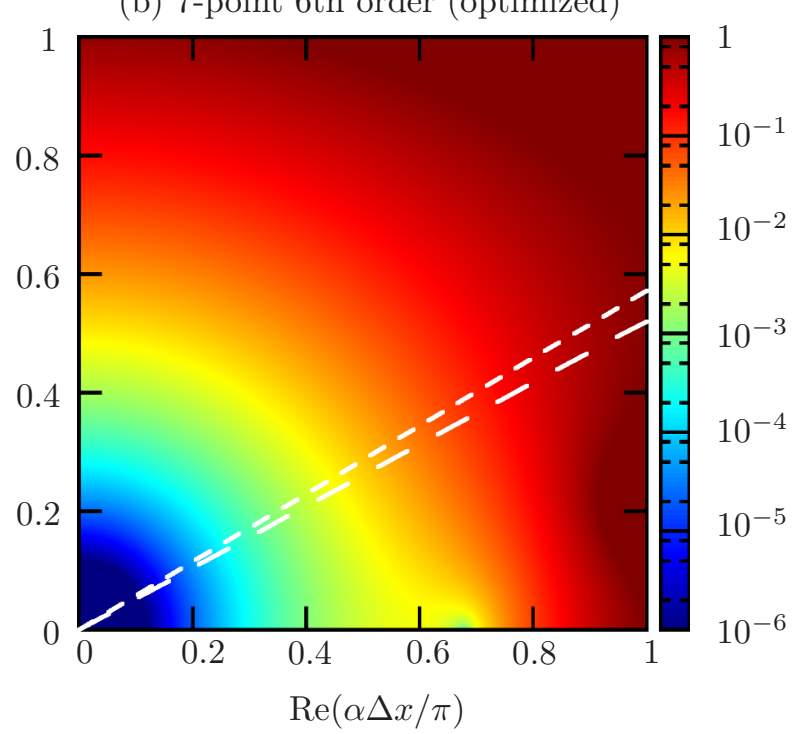

(d) 7-point 4th order (optimized)

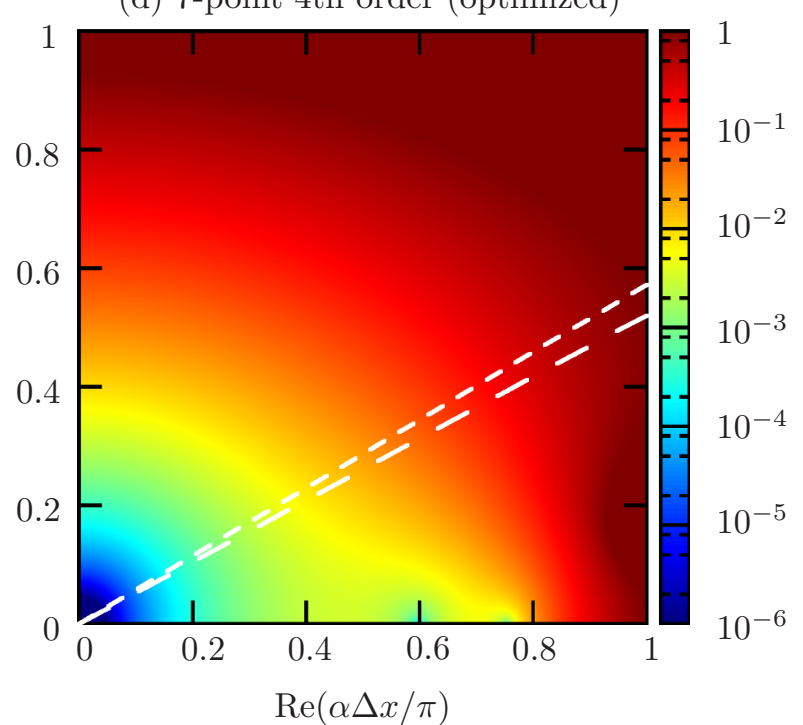

(e) Plots of (a-d) along the short dashed line

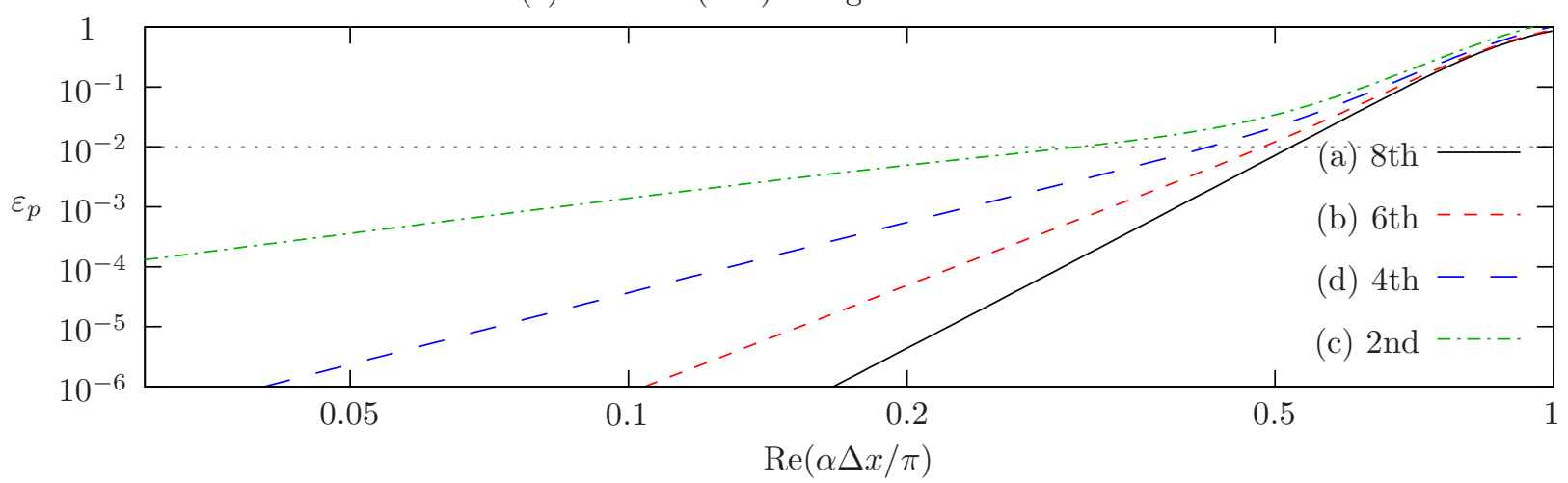

Figure 3: Plots of $\varepsilon_{p}=|\bar{\alpha} / \alpha-1|$ for complex $\alpha$ on a logarithmic scale, giving the relative accuracy of the numerical derivative, for tri-diagonal implicit 7-point schemes from Kim and Lee 8 ; . Short and long dashed lines correspond to $\alpha \Delta x$ for varying $\Delta x$ with and without flow respectively for the axial wavenumbers investigated by Tam et al. [27]. (e) plots the errors of (a-d) along the short dashed line, with the horizontal dotted line indicating a $1 \%$ error. 

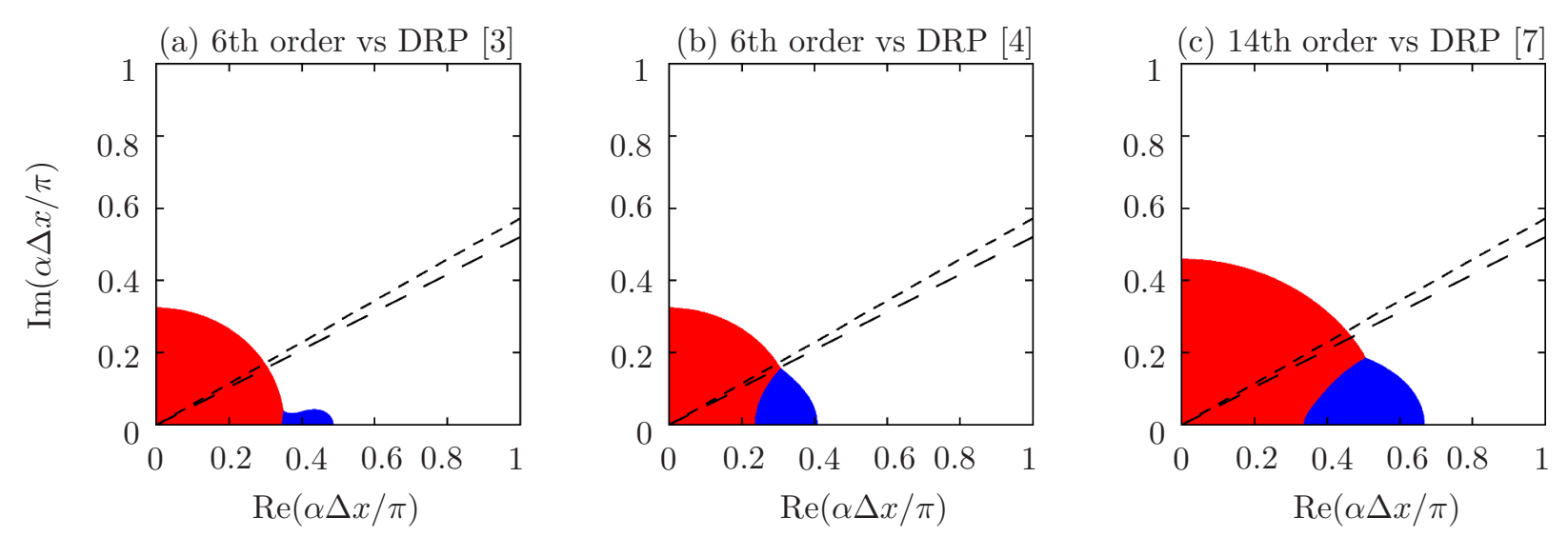

Figure 4: Comparison, for complex wavenumbers $\alpha$, showing where the maximal-order (red) or DRP (blue) scheme is more accurate. White areas indicate regions where neither scheme is within $1 \%$ accuracy $\left(\right.$ i.e. $\left.\varepsilon_{p}=|\bar{\alpha} / \alpha-1|>0.01\right)$. (a) and (b) are 7-point explicitly schemes, while (c) is a 15-point explicit scheme. All DRP schemes are optimized subject to 4th order accuracy. Short and long dashed lines correspond to $\alpha \Delta x$ for varying $\Delta x$ with and without flow respectively for the axial wavenumbers investigated by Tam et al. [27].

(a) 8th order vs 2 nd order

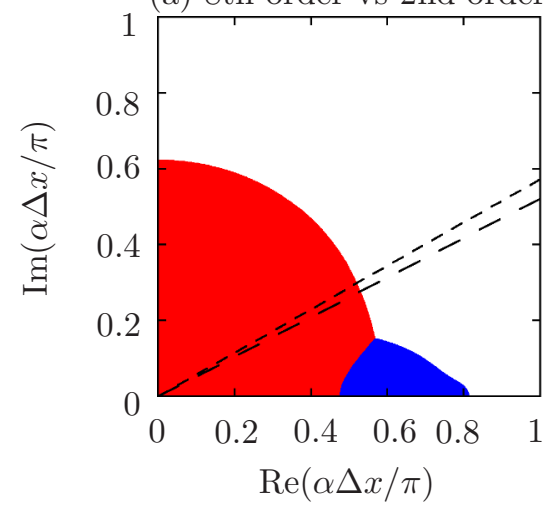

(b) 8th order vs 4th order

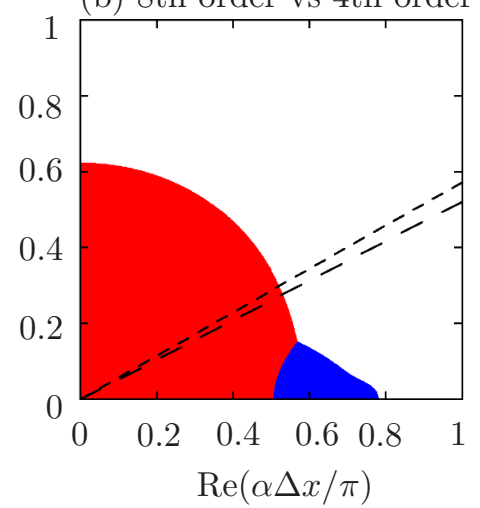

(c) 8th order vs 6 th order

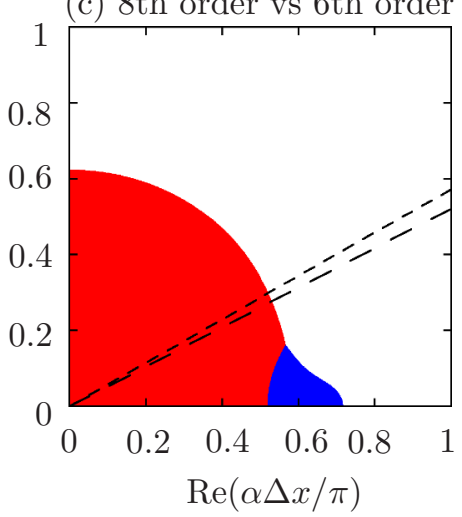

Figure 5: Comparison, for complex wavenumbers $\alpha$, showing which of the maximal-order (8th order, red) or maximum resolution (optimized, blue) 7-point tri-diagonal implicit schemes is more accurate. Optimized schemes are taken from Kim and Lee [8]. White areas indicate regions where neither scheme is within $1 \%$ accuracy (i.e. $\varepsilon_{p}=|\bar{\alpha} / \alpha-1|>0.01$ ). Short and long dashed lines correspond to $\alpha \Delta x$ for varying $\Delta x$ with and without flow respectively for the axial wavenumbers investigated by Tam et al. [27].

axial, radial and azimuthal directions for waves in a cylinder) are known, computational simulations are likely to be more accurate using the classical maximal-order schemes than using optimized DRP schemes.

One obvious class of a priori knowledge would be if waves were known to be of constant amplitude, meaning that $\alpha$ were known to be real. This is unlikely to be true in all direction in practice, but unfortunately it is common to test optimized DRP schemes by solving the $1 \mathrm{D}$ advection equation

$$
\frac{\partial f}{\partial t}+\frac{\partial f}{\partial x}=0
$$

for which waves do propagate with constant amplitude. Other tests also exist, but as well as being less commonly used they rarely involve exponentially rapid decay. This helps explain why flaws in optimized DRP schemes for non-constant-amplitude oscillations have not been demonstrated previously. A more representative test case for investigating non-constant amplitude oscillations is given in the next section. 
(a) 6th order vs DRP [3]

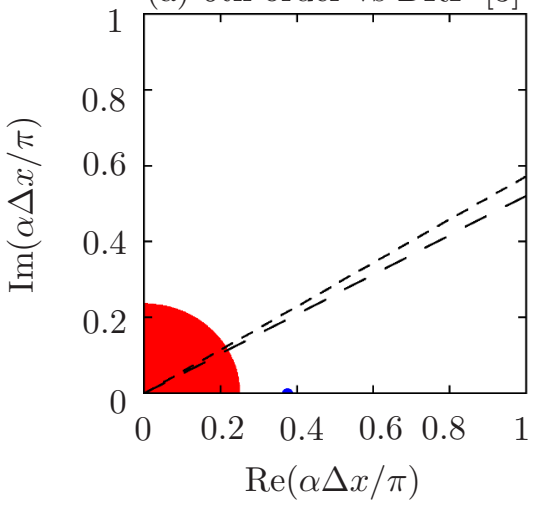

(b) 6th order vs DRP 4

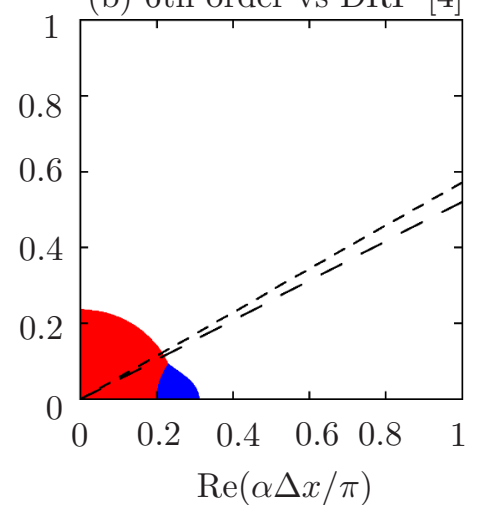

(c) 14 th order vs DRP [7]

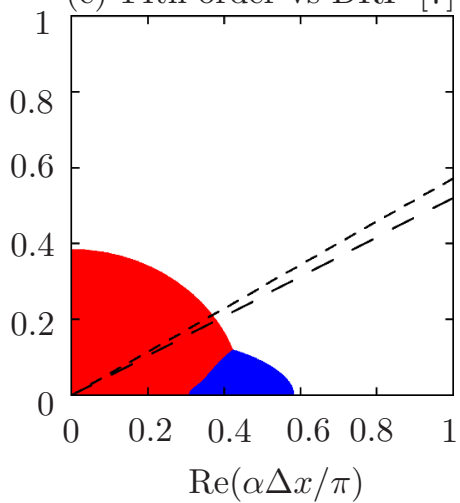

Figure 6: Comparison, for complex wavenumbers $\alpha$, showing where the maximal-order (red) or DRP (blue) scheme is more accurate according to the relative group velocity error $\varepsilon_{g}$. White areas indicate regions where neither scheme is within $1 \%$ accuracy (i.e. $\varepsilon_{g}=|\mathrm{d} \bar{\alpha} / \mathrm{d} \alpha-1|>0.01$ ). (a) and (b) are 7-point explicitly schemes, while (c) is a 15-point explicit scheme. All DRP schemes are optimized subject to 4th order accuracy. Short and long dashed lines correspond to $\alpha \Delta x$ for varying $\Delta x$ with and without flow respectively for the axial wavenumbers investigated by Tam et al. 27].

(a) 8th order vs 2 nd order

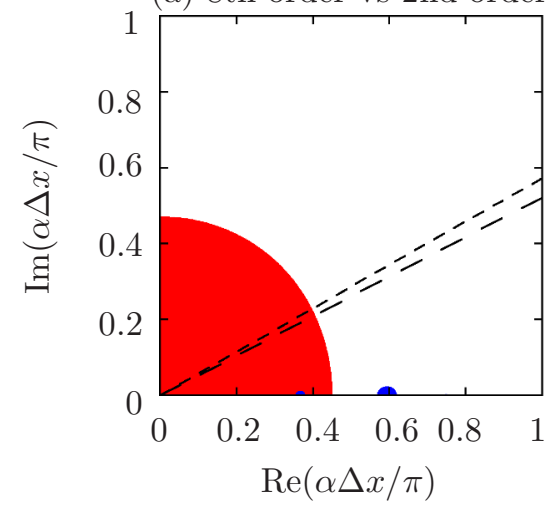

(b) 8th order vs 4th order

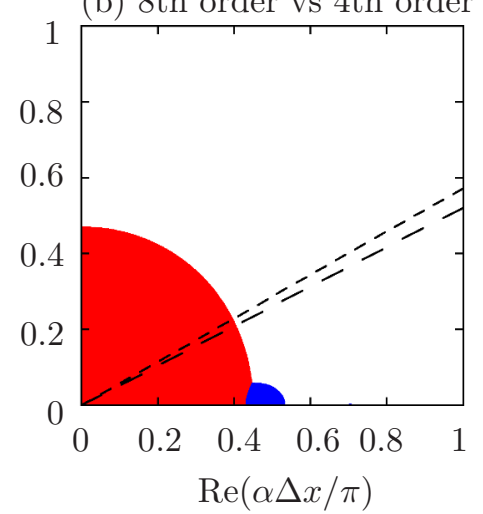

(c) 8th order vs 6th order

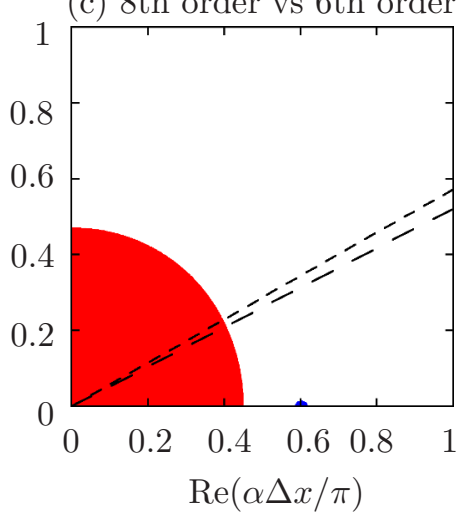

Figure 7: Comparison, for complex wavenumbers $\alpha$, showing which of the maximal-order (8th order, red) or maximal-resolution (optimized, blue) 7-point tri-diagonal implicit schemes is more accurate according to the relative group velocity error $\varepsilon_{g}$. Optimized schemes are taken from Kim and Lee 8]. White areas indicate regions where neither scheme is within $1 \%$ accuracy (i.e. $\varepsilon_{g}=|\mathrm{d} \bar{\alpha} / \mathrm{d} \alpha-1|>0.01$ ). Short and long dashed lines correspond to $\alpha \Delta x$ for varying $\Delta x$ with and without flow respectively for the axial wavenumbers investigated by Tam et al. [27].

\section{Numerical example using the 1D damped wave equation}

In this section, the theoretical points above are illustrated through a $1 \mathrm{D}$ damped wave propagation example. We consider the governing equations

$$
\begin{aligned}
& \frac{\partial p}{\partial t}+\frac{\partial v}{\partial x}=-k_{p}(x) p \\
& \frac{\partial v}{\partial t}+\frac{\partial p}{\partial x}=-k_{v}(x) v
\end{aligned}
$$

where it is emphasized that $p, v, k_{p}$ and $k_{v}$ are all real quantities. These equations support wave propagation in both the positive and negative $x$-directions at a wave speed of 1 . They could be thought of as one dimensional linearized versions of the conservation of mass and conservation of momentum equations of fluid dynamics, with added damping terms $k_{p}$ and $k_{v}$. In this case, the problem could loosely be interpreted as modelling low-frequency sound propagation along a pipe, with the $k_{p}$ pressure damping term thought 


\begin{tabular}{|c|c|c|c|c|c|c|c|}
\hline \multirow[t]{2}{*}{ Derivative } & \multirow[t]{2}{*}{ Width } & \multicolumn{2}{|c|}{$\mathrm{PPCW}$ for $\varepsilon_{p}=10^{-2}$} & \multicolumn{2}{|c|}{$\mathrm{PPCW}$ for $\varepsilon_{p}=10^{-3}$} & \multicolumn{2}{|c|}{ PPW for example } \\
\hline & & Best & Worst & Best & Worst & $1 \%$ & $0.1 \%$ \\
\hline $7 \mathrm{pt} \mathrm{MO}$ & 7 & 5.7 & 6.1 & 8.6 & 8.9 & 14.1 & 20.9 \\
\hline 7pt DRP [3] & 7 & 4.1 & 8.2 & 12.7 & 13.7 & 25.9 & 46.4 \\
\hline 7pt DRP [4] & 7 & 4.9 & 7.2 & 5.9 & 11.6 & 20.4 & 37.2 \\
\hline FDo9p & 9 & 3.8 & 7.2 & 4.2 & 11.2 & 18.5 & 34.3 \\
\hline FDo11p & 11 & 3.5 & 6.8 & 3.9 & 9.8 & 12.6 & 26.0 \\
\hline FDo13p & 13 & 3.1 & 7.5 & 3.4 & 10.8 & 14.1 & 28.8 \\
\hline 15pt MO & 15 & 3.6 & 4.3 & 4.4 & 5.0 & 6.8 & 8.2 \\
\hline 15pt DRP [7] & 15 & 3.0 & 7.3 & 3.3 & 10.1 & 7.2 & 23.4 \\
\hline KL2 & t7 & 2.5 & 8.0 & 2.5 & 21.6 & 80.0 & 253.0 \\
\hline KL4 & $\mathrm{t} 7$ & 2.6 & 4.9 & 2.6 & 8.1 & 14.8 & 26.8 \\
\hline KL6 & t7 & 2.8 & 3.8 & 2.9 & 5.4 & 8.4 & 12.7 \\
\hline KL8 (MO) & $\mathrm{t} 7$ & 3.2 & 3.4 & 4.3 & 4.5 & 7.2 & 9.3 \\
\hline
\end{tabular}

Table 1: Resolution (in PPCW $=2 \pi /|\alpha \Delta x|$ ) needed to achieve a given accuracy. Width gives the width of the schemes in points, with "t7" indicating a 7-point tridiagonal schemes. Best and worst refer to varying $\arg (\alpha \Delta x)$. The "PPW for example" columns give the resolution needed to achieve a given accuracy for the example problem of section 3 after the accumulation of error over a large number of time steps, with "perfect" spatial filtering and temporal integration.

\begin{tabular}{l|ccc} 
& $\begin{array}{c}\text { Liner length in } \\
\text { axial wavelengths }\end{array}$ & $\begin{array}{c}\text { overall SPL } \\
\text { reduction }\end{array}$ & $\begin{array}{c}\text { SPL reduction } \\
\text { per axial wavelength }\end{array}$ \\
\hline [27], no flow & 1.12 & $-31.7 \mathrm{~dB}$ & $-28.2 \mathrm{~dB}$ \\
[27], with flow & 1.60 & $-50.0 \mathrm{~dB}$ & $-31.2 \mathrm{~dB}$ \\
Here & $\approx 2$ & $-52.1 \mathrm{~dB}$ & $-26.1 \mathrm{~dB}$
\end{tabular}

Table 2: Comparison (in non-dimensional terms) between the industrially motivated parameters used by Tam et al. 27] and the parameters used here for the example wave-propagation problem.

of as a leaky pipe loosing or gaining fluid proportional to the perturbation pressure $p$, and the $k_{v}$ velocity damping thought of as a drag term proportional to the axial perturbation velocity $v$. For our purposes here, however, (7) is just regarded as a set of equations to be solved numerically.

For the special case $k_{p}(x)=k_{v}(x)$ and with the special initial conditions $v(x, 0)=p(x, 0)=p_{0}(x)$, equation (7) admits the analytic solution

$$
v(x, t)=p(x, t)=p_{0}(x-t) \exp \left\{-\int_{x-t}^{x} k_{p}(X) \mathrm{d} X\right\},
$$

which represents a right-propagating wave with an amplitude that decays according to the cumulative damping seen during its propagation.

Here, we consider a periodic $x$-domain $[0,24)$, initial conditions consisting of a wave packet with wavelength 1 occupying $x \in[2,18]$, and a region of damping of strength $k_{p}=k_{q}=3$ occupying $x \in[21,23]$. This gives a complex wavenumber $\alpha=2 \pi-3 \mathrm{i}$ in the damped region and $\alpha=2 \pi$ elsewhere. Because of these two wavenumbers, all results will be given here in terms of points per (real) wavelength, $\mathrm{PPW} \equiv 2 \pi / \operatorname{Re}(\alpha \Delta x)=1 / \Delta x$. This example problem is intended to test simultaneously both wave propagation with significant damping per wavelength and undamped wave propagation over many wavelengths (22 wave lengths in this case). The coefficients of this example problem have been inspired by the study of Tam et al. 27] excluding the effects of splices, as shown in table 2. In particular, the amount of damping, the distance over which the damping is applied, and the axial wavelengths are all broadly comparable. As mentioned previously, Tam et al. 27] showed that the presence of liner splices could scatter the incoming wave into other waves with an amplitude of $1 \%$ of that of the incident wave, and that these other waves are less well attenuated and therefore become dominant by the end of the liner, and so at least $1 \%$ accuracy will be required here. 
In order to allow high-order schemes to achieve their potential, the initial conditions and damping need to be made sufficiently smooth. Hence, the amplitude of the wave packet is modified to vary smoothly for $x \in(0,4)$ and $x \in(16,20)$. Similarly, the damping is modified to vary smoothly for $x \in\left(20 \frac{1}{2}, 21 \frac{1}{2}\right)$ and $x \in\left(22 \frac{1}{2}, 23 \frac{1}{2}\right)$. The exact formula for the initial conditions and damping profile are given by

$$
v(x, 0)=p(x, 0)=\cos (2 \pi x) B(x ; 0,4,16,20,8), \quad k_{v}(x)=k_{p}(x)=3 B\left(x ; 20 \frac{1}{2}, 21 \frac{1}{2}, 22 \frac{1}{2}, 23 \frac{1}{2}, 8\right),
$$

where $B$ is a relatively smooth piecewise-polynomial bump function given by

$$
B(x ; a, b, c, d, n)=\left\{\begin{array}{cl}
0 & \text { if } x \leq a \\
\left(\frac{x-a}{b-a}\right)^{n} \sum_{j=0}^{n-1}\left(\begin{array}{c}
n+j-1 \\
j
\end{array}\right)\left(\frac{b-x}{b-a}\right)^{j} & \text { if } a<x<b \\
1 & \text { if } b \leq x \leq c \\
\left(\frac{d-x}{d-c}\right)^{n} \sum_{j=0}^{n-1}\left(\begin{array}{c}
n+j-1 \\
j
\end{array}\right)\left(\frac{x-c}{d-c}\right)^{j} & \text { if } c<x<d \\
0 & \text { if } x \geq d
\end{array}\right.
$$

This initial situation is shown in figure 8(a). After a time $t=12$, the wave will have propagated half way across the periodic $x$-domain, and the part that has crossed the damping will have decayed. This is depicted in figure 8 (b) using a linear scale, and in figure 8(c) using a logarithmic scale. At time $t=24$, the initial wave should have propagated exactly once around the periodic $x$-domain. Noting that $\int_{a}^{d} B(x ; a, b, c, d, n) \mathrm{d} x=$ $\frac{c+d}{2}-\frac{a+b}{2}$, at time $t=24$ the wave will also have decayed by a factor of $\mathrm{e}^{-6} \approx 1 / 400$, or $-52.1 \mathrm{~dB}$ SPL. Hence, we define the error in the solution at time $t=24$ as

$$
E=\sup _{x \in[0,24)}\left\{\left|p_{0}(x)-\mathrm{e}^{6} p(x, 24)\right|,\left|p_{0}(x)-\mathrm{e}^{6} v(x, 24)\right|\right\}
$$

We investigate the resolution required to achieve $E=0.01$, in line with the $1 \%$ error requirement mentioned above.

\subsection{Testing spatial derivatives only}

First of all, we investigate the effect of only the spatial derivatives on numerical solutions to this onedimensional wave propagation problem. To do this, we introduce "perfect" spatial filtering and time integration, where "perfect" means that the errors due to spatial filtering and time integration are negligible when compared to the errors due to the spatial differentiation. To do this, the following results use a 4 th order adaptive timestep Runge-Kutta temporal integration (RK45) 30] with an error tolerance of $10^{-8}$ and a 19-point spatial filter with a 16 th order error labelled $F_{16,4}$ (the details of which are given in appendix A). The filter is applied once at each time step with a strength $S \Delta t$, with $S$ chosen to optimize the results for each spatial derivative. The values of $S$ used are given in table A.4.

Figure 9] shows the variation of error (given by equation[11) with the number of points per real wavelength for several different spatial schemes. The FDo9p, FDo11p and FDo13p are the optimized spatial derivatives proposed by Bogey and Bailly [14], while all the other DRP schemes have 4th order accuracy. Unsurprisingly the wider schemes, which require more computational effort per derivative (see section 3.3), perform better with fewer points per wavelength, but for any given width the scheme to achieve the desired $1 \%$ error for the fewest points per wavelength is seen to be the maximal-order scheme. If a stricter error tolerance were required, such as $0.1 \%$ error, the maximal-order schemes are better by a wider margin compared with optimized schemes. This is unsurprising given the results of figures 2 and 4 above. The number of points per wavelength required to achieve a given accuracy for this example are also listed in table 1 above.

If we consider implicit differentiation schemes, the picture is rather similar, as shown in figure 10. Of the 7 -point tri-diagonal schemes, the one to reach $1 \%$ accuracy for the fewest points per wavelength is the maximal-order (8th order) scheme labelled KL8 (as predicted by figures 3 and 5 earlier). The 4th order 
(a) $t=0$

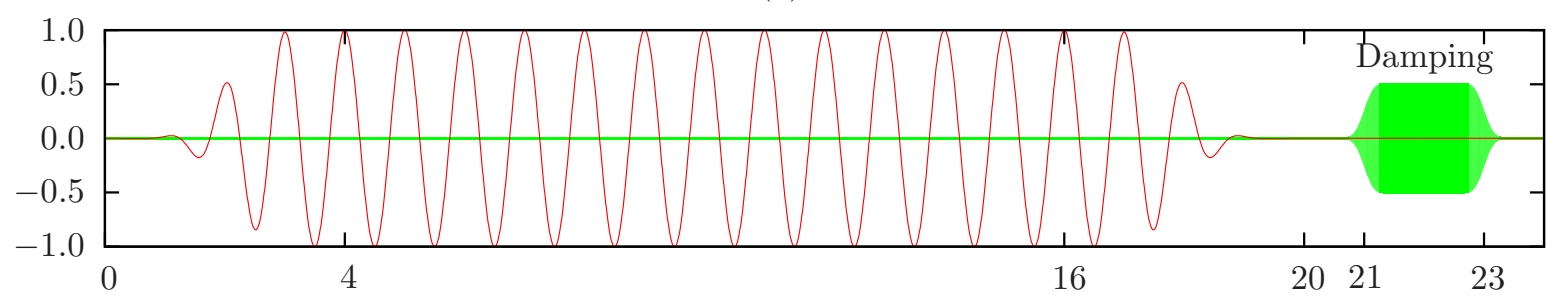

(b) $t=12$

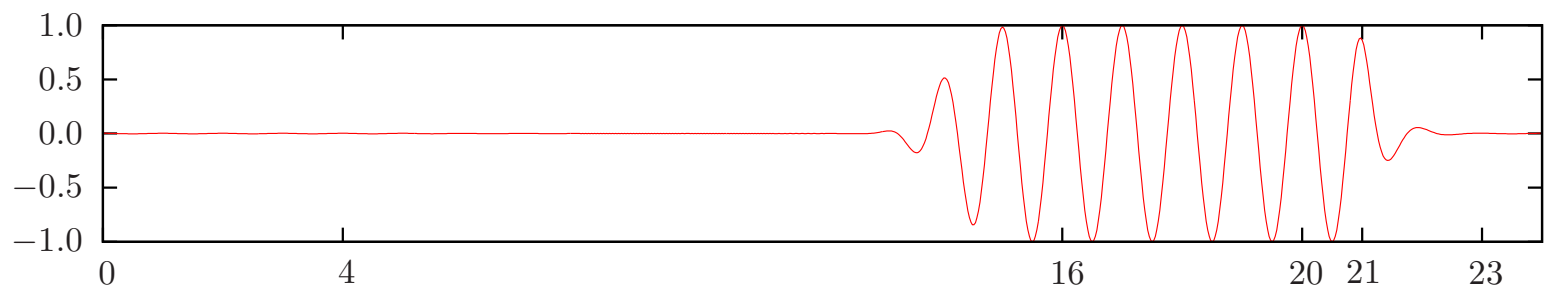

(c) $t=12($ logarithmic scale)

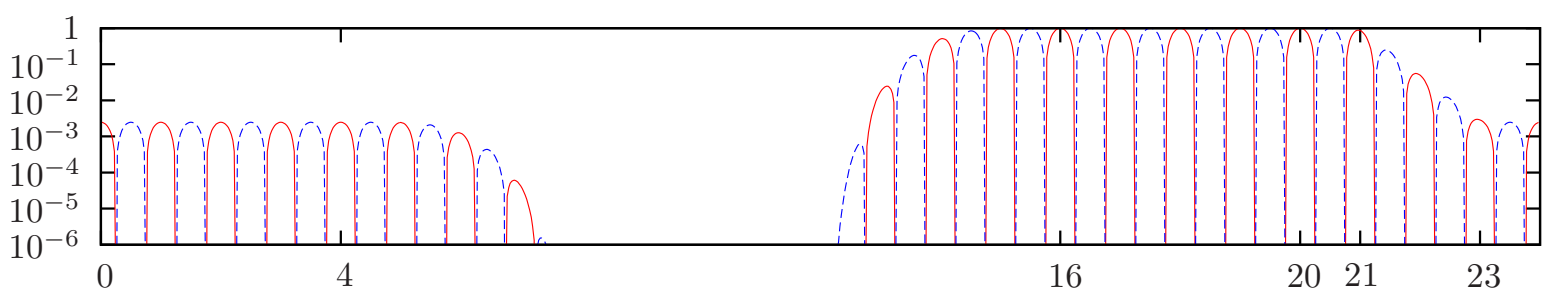

Figure 8: Diagram of the wave problem considered. The domain is periodic in the $x$-direction. The initial wave in (a) has amplitude 1 for $x \in[4,16]$ and smoothly decays to zero amplitude for $x \in(0,4)$ and $x \in(16,20)$. The damping (rescaled by $1 / 6$ ) in (a) has equivalent length 2 , with full amplitude for $x \in\left[21 \frac{1}{2}, 22 \frac{1}{2}\right]$ and a smooth decay to zero amplitude for $x \in\left(20 \frac{1}{2}, 21 \frac{1}{2}\right)$ and $x \in\left(22 \frac{1}{2}, 23 \frac{1}{2}\right)$. (b) and (c) show the wave at time $t=12$ on a linear (b) or logarithmic (c) scale. In (c), dashed lines denote negative values and solid lines denote positive values. An animation of this figure is given in the supplementary material.

optimized scheme KL4 of Kim and Lee [8] is seen in this case to require more points per wavelength than the explicit maximal-order scheme labelled MO7. These results are also summarized in table 1 above. It should be noted that computing the implicit derivatives for any of the 7-point tri-diagonal schemes took over 14 times longer than computing the 7-point explicit schemes; computational effort will be discussed further in section 3.3 ,

It is clear from these figures that the conclusions of section 2 that optimized schemes perform poorly for non-constant-amplitude oscillations are supported by this example wave-propagation problem. However, it remains to be seen whether this difference in accuracy impacts the overall accuracy of simulations when more commonly used spatial filters and time integrators are used, rather than the "perfect" filters and integrators used here. We consider this in the next section.

\subsection{Accuracy of derivatives in practice}

Figure 11 shows the effect of varying the time integration used, both for 7-point spatial derivatives with 7-point spatial filters and for 15-point spatial derivatives with the high-order $F_{16,4}$ filter used previously. The widely used LDDRK56 optimized 4th order time integration scheme by Hu, Hussaini, and Manthey [31] and the RKo6s optimized time integration scheme by Bogey and Bailly [14] are used for this comparison, with timesteps $\Delta t$ given by CFL numbers of 0.2 and 0.8 , where CFL $=\Delta t / \Delta x$ for this example. As expected, 


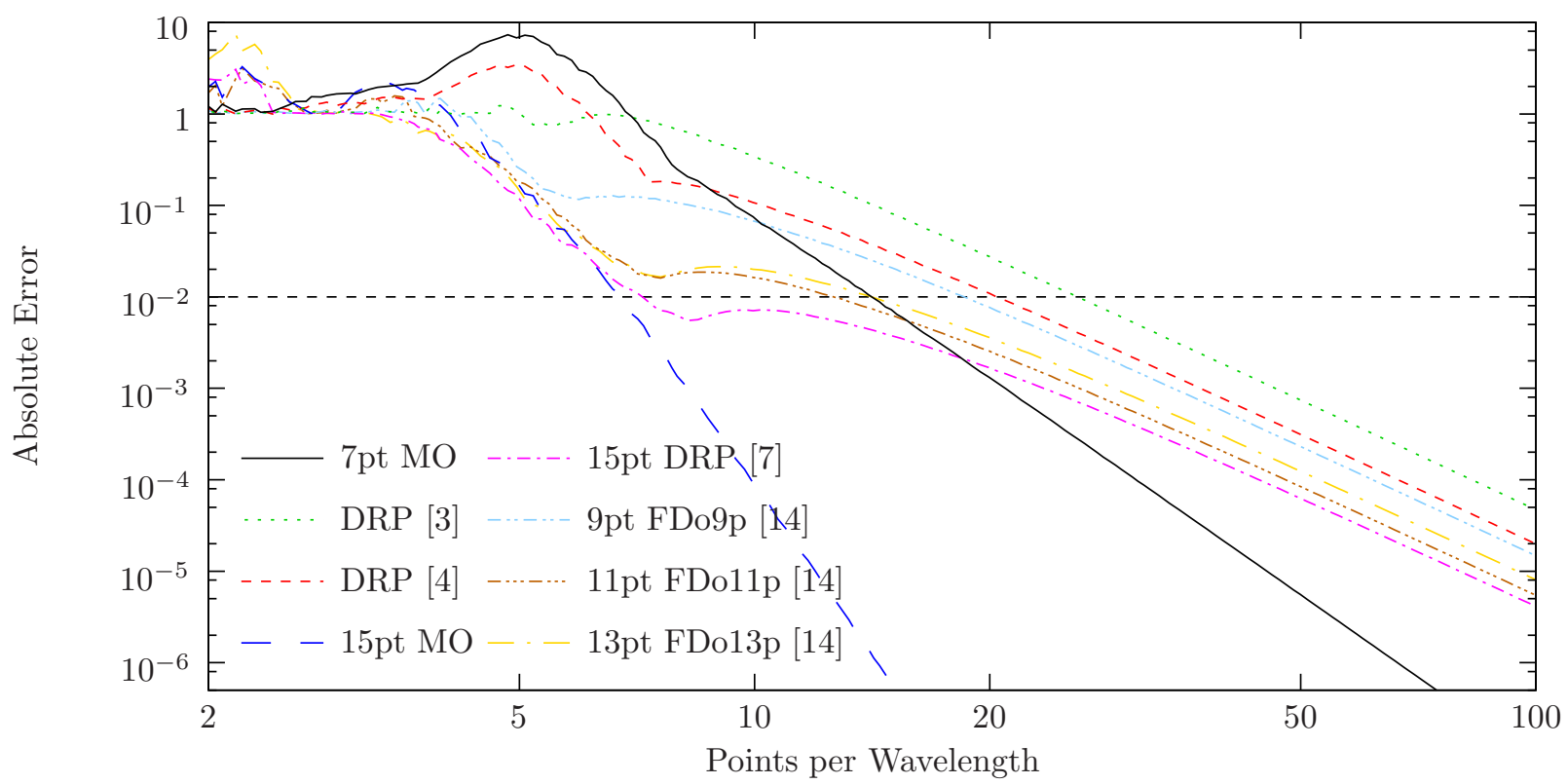

Figure 9: Absolute error against points per wavelength for several explicit spatial derivative schemes, all using the high-order 19-point $F_{16,4}$ filter and RK45 adaptive time stepping.

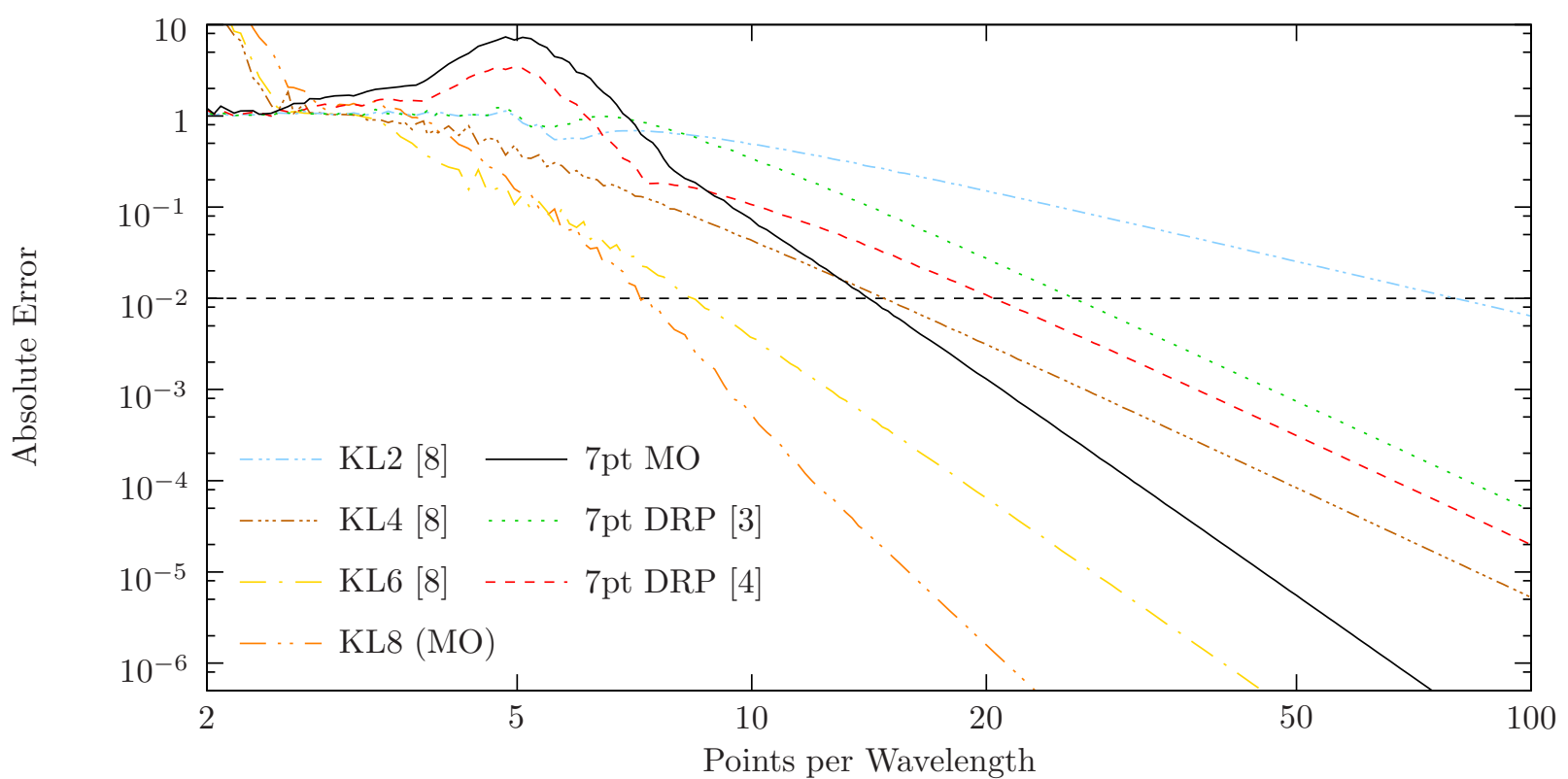

Figure 10: Absolute error against points per wavelength for several 7-point spatial derivative schemes, all using the high-order 19-point $F_{16,4}$ filter and RK45 adaptive time stepping. KL2-KL8 denote the 2nd-8th order Kim and Lee [8] tri-diagonal schemes, with KL8 being the maximal-order scheme and the others being optimized schemes. 
(a) 7-point $\mathrm{MO}$

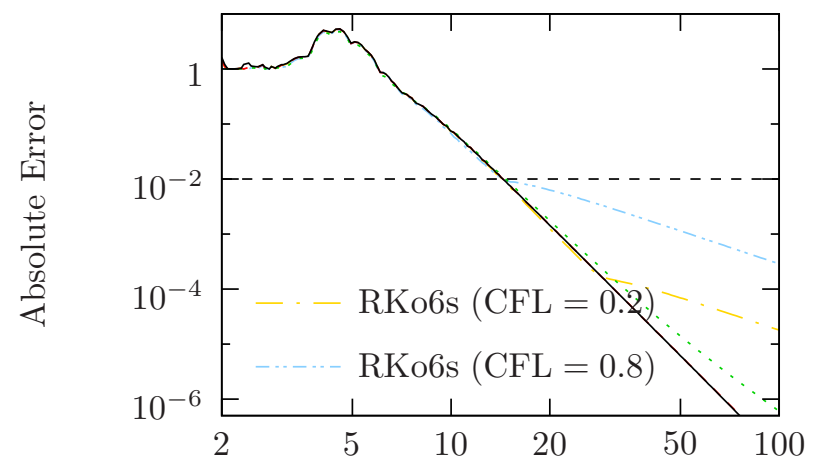

(c) 15-point $\mathrm{MO}$

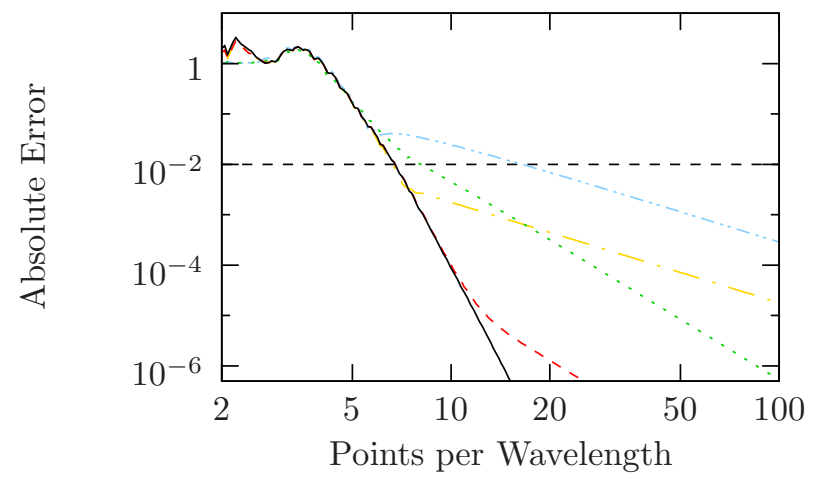

(b) 7-point DRP [4]

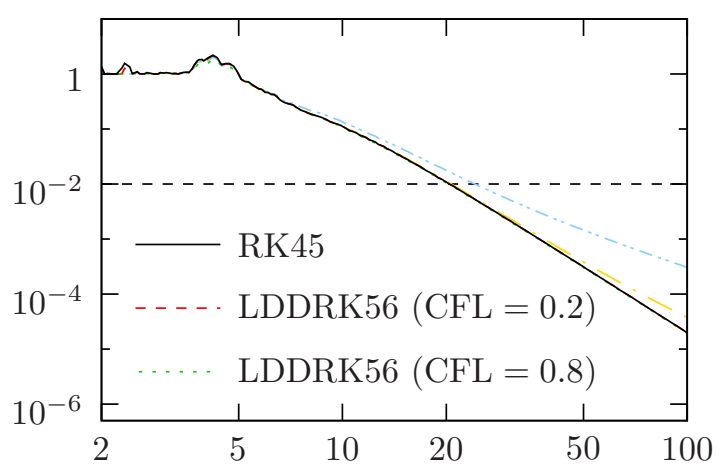

(d) 15-point DRP [7]

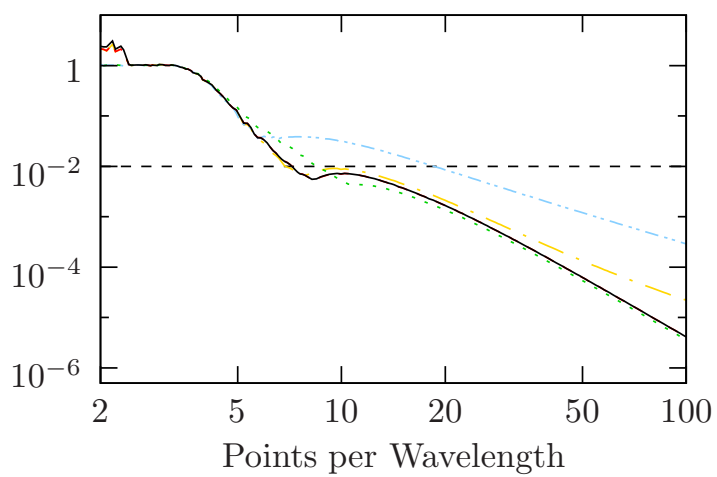

Figure 11: Absolute Error against points per wavelength for several time stepping schemes. (a) and (b) use 7-point spatial derivatives and the 7-point 6th-order filter $F_{6}$, while (c) and (d) use 15-point spatial derivatives and the 19-point high-order filter $F_{16,4}$.

for few points per wavelength the error is dominated by the spatial integration, while for many points per wavelength the wave is well resolved spatially and so the time integration error dominates. Provided the damping strength $S$ is chosen correctly and the filter is of sufficiently high order, the spatial filter used is never the limiting factor in this example.

Figure 12 compares a number of different schemes typical of those used in practice, as well as two higherorder 15-point schemes. The most commonly used of all these is probably the DRP spatial derivative of Tam and Shen [4] with the 7-point 6 th order $F_{6}$ spatial filter and the LDDRK56 optimized time integration scheme. Plotted also are the 9, 11, and 13 point optimized spatial derivatives and filters of Bogey and Bailly [14, all using the 6-step optimized Runge-Kutta time integration proposed by the same authors. The number of points per (real) wavelength needed to achieve the desired $1 \%$ error tolerance at a CFL number of 0.8 is listed in table 3 . In summary, for this example there is no advantage to using an optimized scheme over a maximal-order scheme, and in many cases there is a significant detriment in doing so.

As yet we have given little consideration to the computational effort. However, provided computational memory is not the limitation, a lower-order scheme could provide better accuracy for the same computational effort despite requiring more points per wavelength than a higher-order scheme. We now investigate this further.

\subsection{Accuracy vs computational effort}

Under the assumption that neither memory nor cache bandwidth are the limiting factor in an optimized solver, an indication of computational effort is given by the number of addition and multiplication operations needed to evaluate the numerical schemes. If the spatial derivative has width $m$ points, the spatial filter 
(a) $\mathrm{CFL}=0.2$

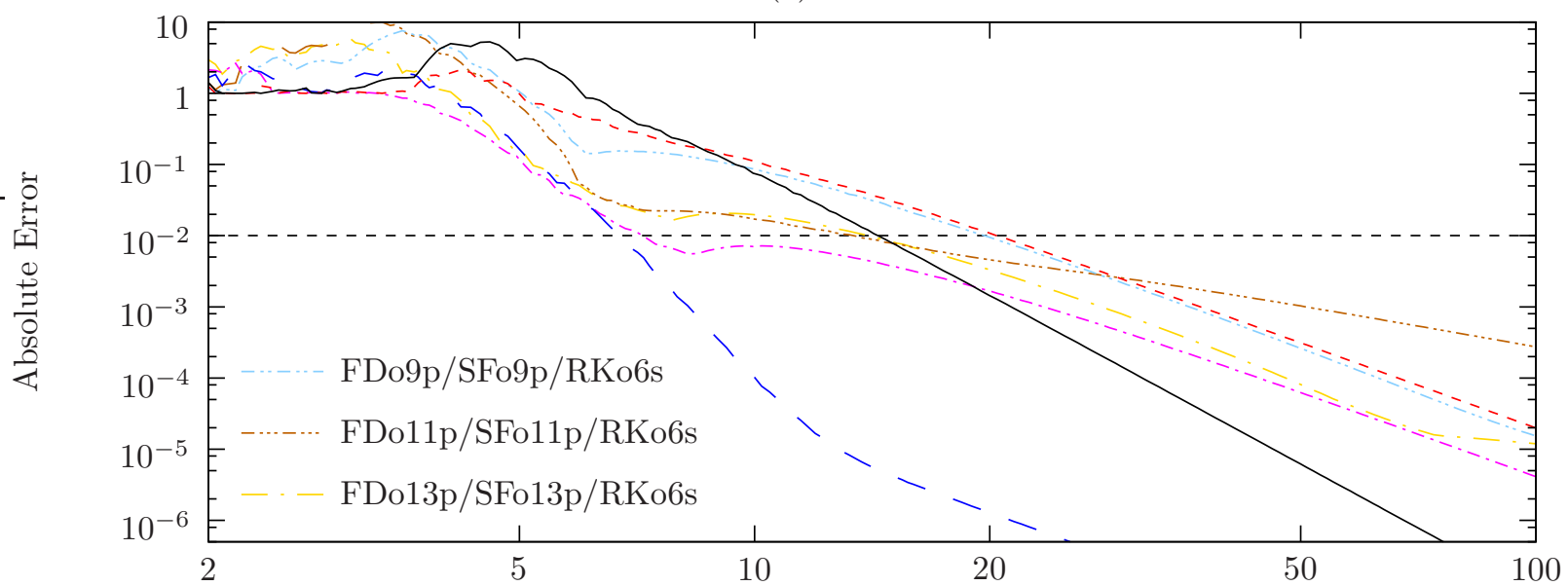

(b) $\mathrm{CFL}=0.8$

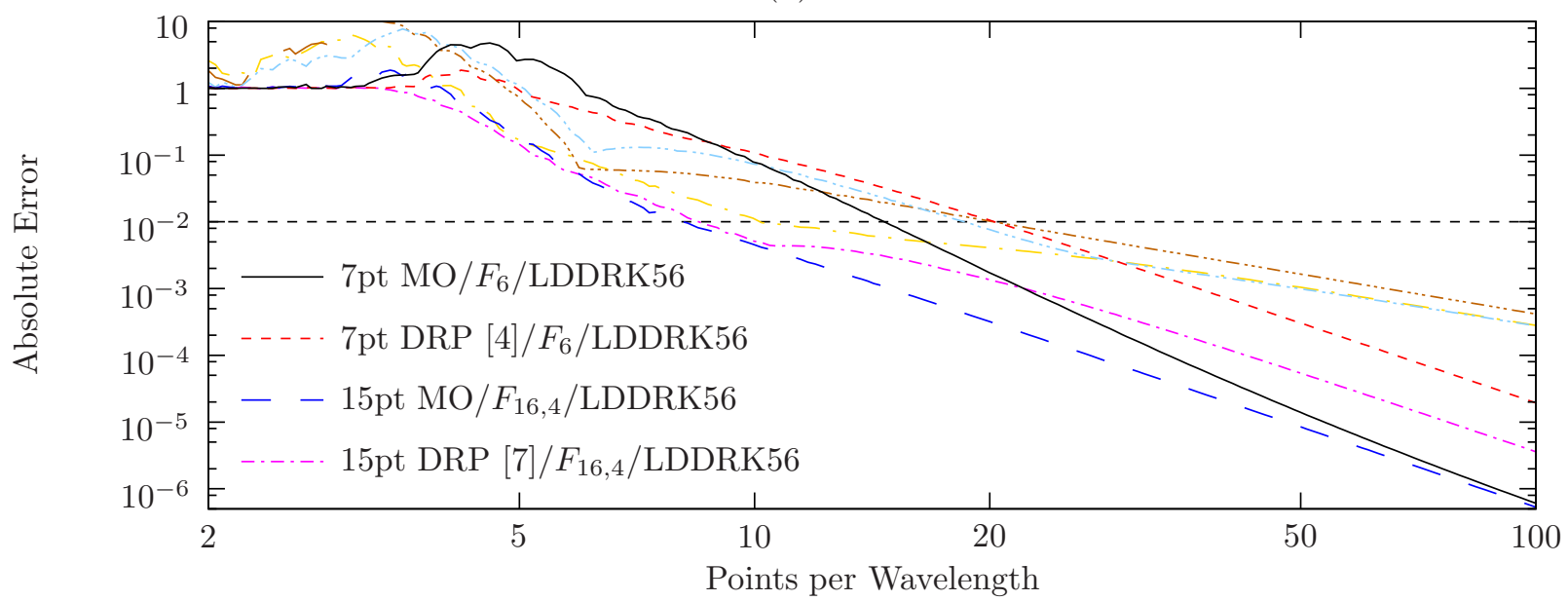

Figure 12: Comparison of the errors of different overall schemes. Curves are labelled spatial scheme/filter/time integrator, with FDo9p, FDo11p and FDo13p being the optimized schemes of Bogey and Bailly [14].

\begin{tabular}{lrrrrrrr} 
Derivative & Filter & Time integrator & CFL & \multicolumn{2}{c}{$1 \%$ error } & \multicolumn{2}{c}{$0.1 \%$ error } \\
& & & & PPW & Effort $/ 10^{3}$ & PPW & Effort $/ 10^{3}$ \\
\hline 15pt MO & $F_{16,4}$ & LDDRK56 & 0.8 & 8.1 & 11.3 & 14.9 & 38.2 \\
15pt DRP [7] & $F_{16,4}$ & LDDRK56 & 0.8 & 8.5 & 12.4 & 22.1 & 84.4 \\
7pt MO & $F_{6}$ & LDDRK56 & 0.8 & 14.7 & 14.5 & 22.1 & 33.0 \\
FDo13p & SFo13p & RKo6s & 0.8 & 10.2 & 15.6 & 51.1 & 395.4 \\
7pt DRP [4] & $F_{6}$ & LDDRK56 & 0.8 & 20.3 & 27.7 & 37.0 & 92.2 \\
FDo9p & SFo9p & RKo6s & 0.8 & 18.5 & 33.9 & 49.7 & 244.2 \\
FDo11p & SFo11p & RKo6s & 0.8 & 20.2 & 51.2 & 64.3 & 516.4
\end{tabular}

Table 3: Computational schemes ordered by effort to achieve 1\% accuracy, together with the points per (real) wavelength and computational effort needed to achieve the given error tolerances. For each scheme, the result is for the best set of parameters considered here. 


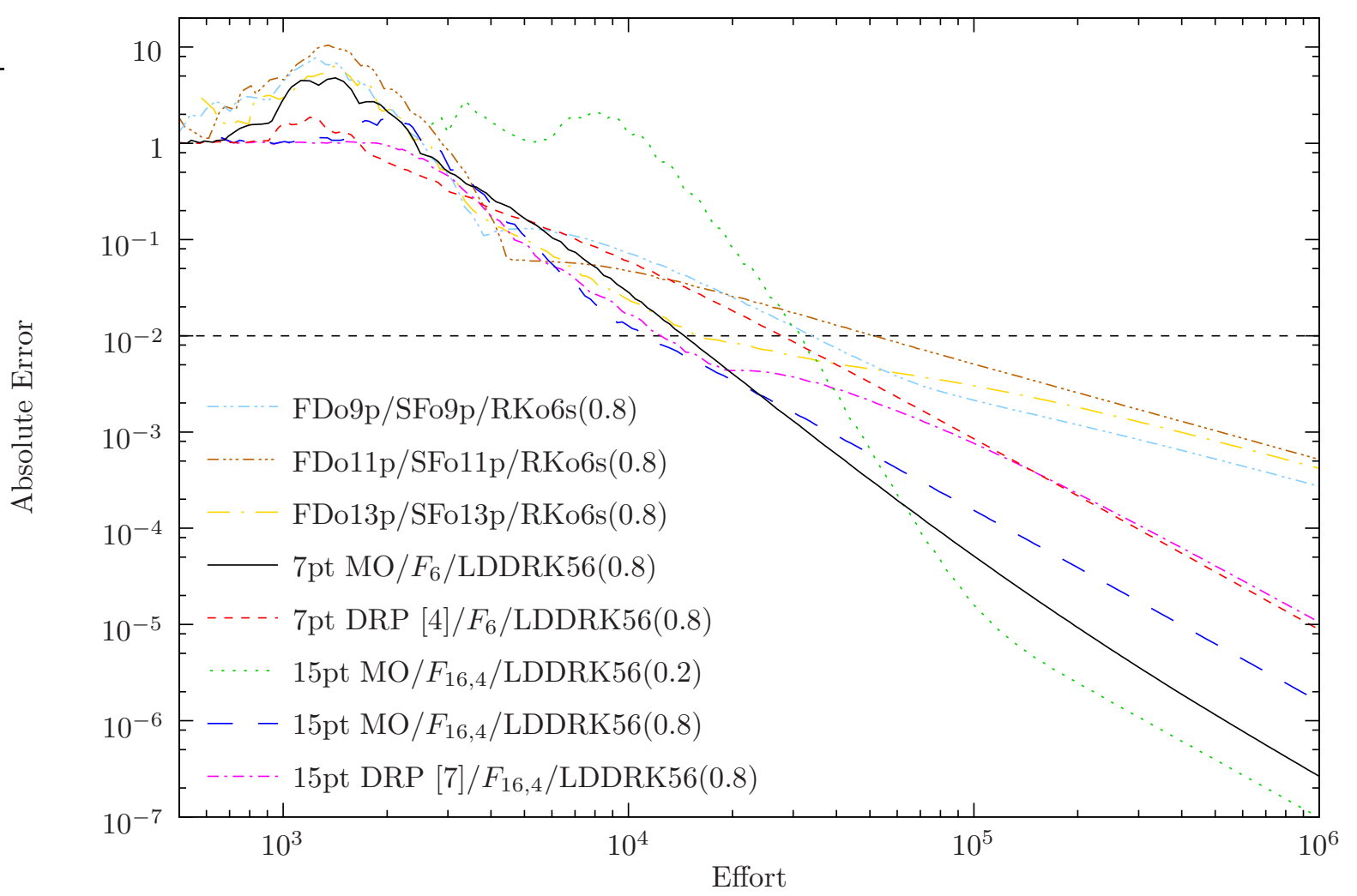

Figure 13: Comparison of the errors of different overall schemes against the computational effort needed. Curves are labelled spatial scheme/filter/time integrator(CFL). Effort is the number of multiplication and addition operations needed per wavelength per wave period given by 12 .

width $n$ points, and the time integrator uses $r$ evaluations per time step, then operating at a given number of points per wavelength PPW and a given CFL number, the effort in solving (7) per wavelength per wave period, is given by

$$
\text { Effort }=\frac{\mathrm{PPW}^{2}}{2 \mathrm{CFL}}(r(3 m-5)+3 n-1)
$$

Figure 13 plots the numerical effort against accuracy for the schemes from figure 12 that give the best accuracy for a given amount of effort. For a desired error of 1\%, the best schemes are the 15-point maximalorder, followed by the 15-point 4th order DRP and the 7-point maximal-order, while for $0.1 \%$ error the best schemes are the 7-point maximal-order followed by the 15-point maximal-order. The required effort is also listed in table 3. Note that, for a desired error of 1\%, the optimized 7-point DRP scheme of Tam \& Shen requires $191 \%$ the computational effort of the 7-point maximal-order scheme to achieve the same precision. The advantage of the maximal-order schemes only increases if a smaller error is required (for example, to achieve $0.1 \%$ accuracy the optimized 7 -point DRP scheme of Tam \& Shen requires $279 \%$ the effort of the 7-point maximal-order scheme).

Numerical tests suggest that the 7-point implicit tri-diagonal schemes are 14.2 times slower to compute than their 7-point explicit counterparts. This can be incorporated into (12) by artificially setting $m=77$ for these implicit schemes. Doing so produces figure 14, demonstrating that, at least for this example problem, implicit schemes of any sort are uncompetitive when compared with explicit schemes. Of course, the real advantage of implicit schemes is when a matrix solve is already required, such as in the frequency domain when considering eigenvalue or boundary value problems.

It should also be noted that the greater simplicity of 7-point schemes when compared with wider schemes, and the requirement of greater smoothness by higher-order schemes to achieve their potential, means that 


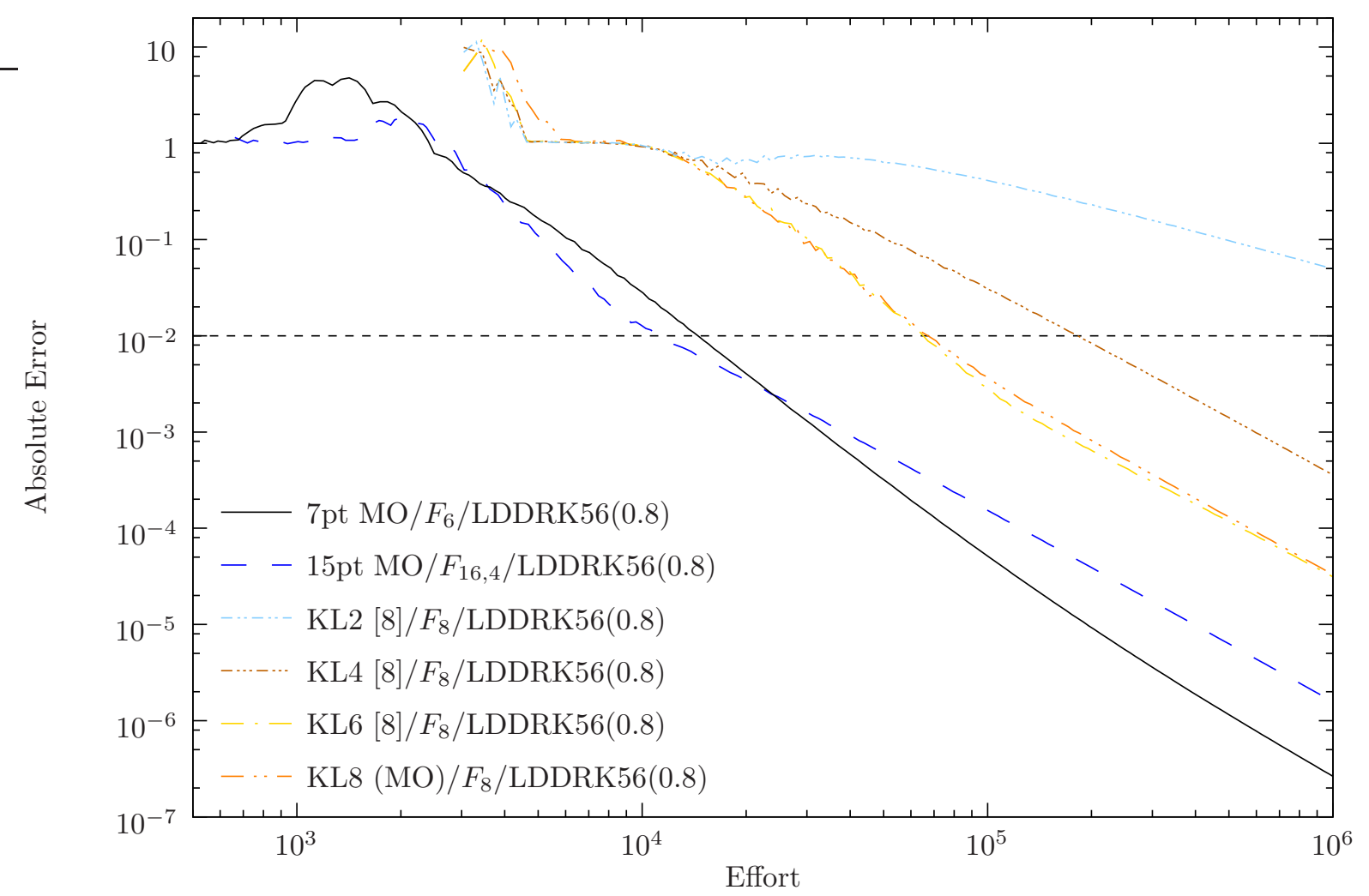

Figure 14: Comparison of the errors of different overall schemes against the computational effort needed. Curves are labelled spatial scheme/filter/time integrator(CFL). Effort is given by (12), with the artificial width of $m=77$ for the KL schemes determined empirically to represent the effort required in solving for the derivatives for these implicit schemes.

a 7-point scheme may well be preferable even if it requires slightly more computational effort. For this and similar 1D problems, therefore, the author recommends the 7-point maximal-order scheme in combination with a 7 -point maximal-order filter $\left(F_{6}\right)$ and the LDDRK56 optimized time integration at a CFL number of 0.8 , with around 15 points per (real) wavelength, or 14 points per complex wavelength. Of course, for wave equations with two or more spatial dimensions, the effort estimate in (12) will have a different scaling, being proportional to $\mathrm{PPW}^{D+1}$ in $D$ dimensions, and a separate analysis would be needed to find the most efficient width, although this would not affect the relative performance of maximal-order schemes compared with optimized schemes of the same width.

\subsection{Synergy between spatial and temporal schemes}

During the course of this work, it was observed that certain combinations of temporal and spatial schemes were able to perform better together than would be expected from their individually analysed performance when using the rather basic fixed time step RK4 time integration. As an example, figure 15 shows that using an RK4 temporal integrator at $\mathrm{CFL}=0.8$ produces better results for the 7-point DRP scheme of Tam \& Shen than using either the LDDRK56 or the RK45 adaptive time step integrators (although even this RK4 combination does not outperform the 7-point maximal-order scheme). This effect is certainly not universal, as both the 7-point maximal-order and the optimized KL4 spatial integrals perform worse using RK4 than when using LDDRK56. Because the LDDRK56 results are the same for both 0.2 and 0.8 CFL numbers, which both agree with the RK45 adaptive integrator results, the error must be dominated by the spatial derivative in this case, and hence it is concluded that there is either beneficial or detrimental synergy between the spatial and temporal operators. This interesting effect is not pursued further here, and it is noted that while certain schemes (such as the CABARET scheme of Karabasov and Goloviznin 32, 33]) combine 
(a) $7 \mathrm{pt} \mathrm{MO}$

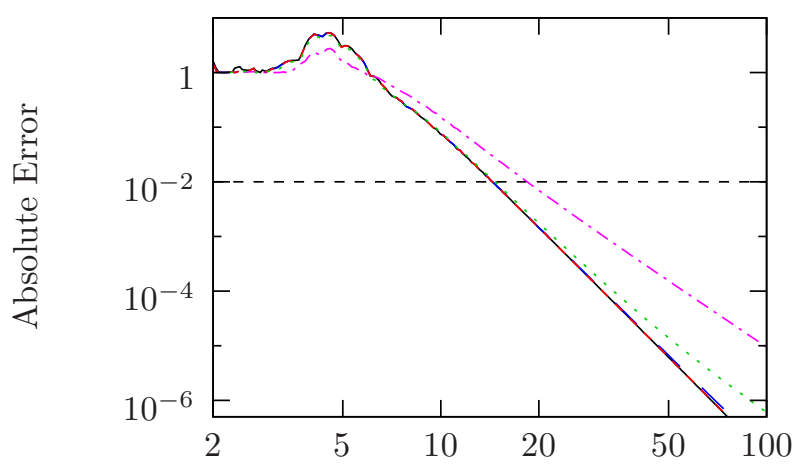

(c) KL2: 7pt 2nd order optimize implicit [8]

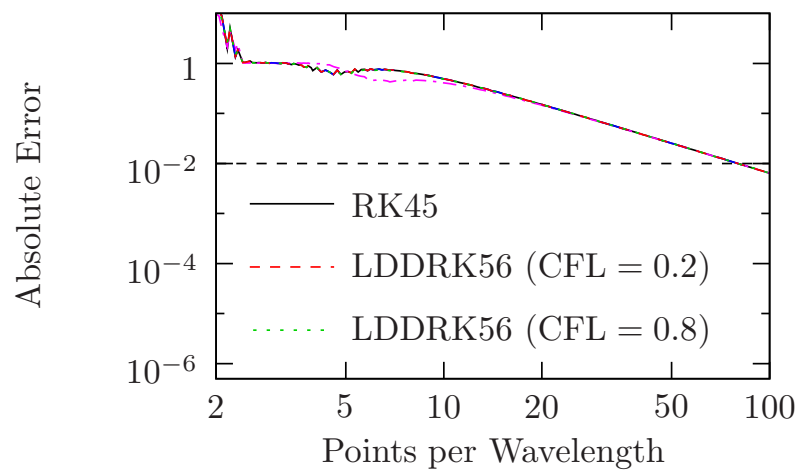

(b) $7 \mathrm{pt}$ DRP [4]

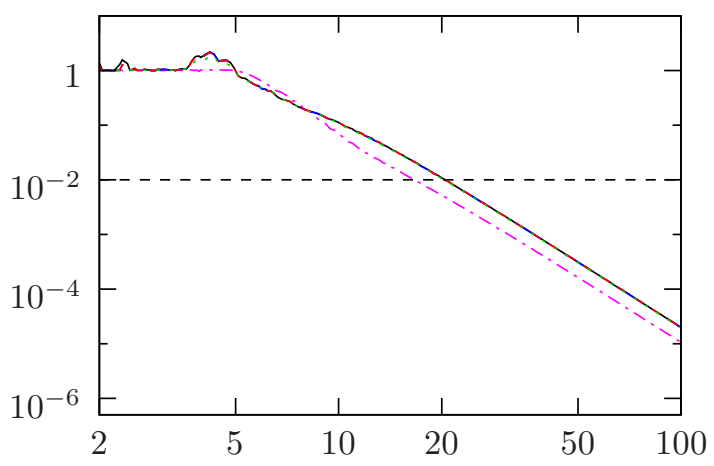

(d) KL4: 7pt 4th order optimize implicit [8]

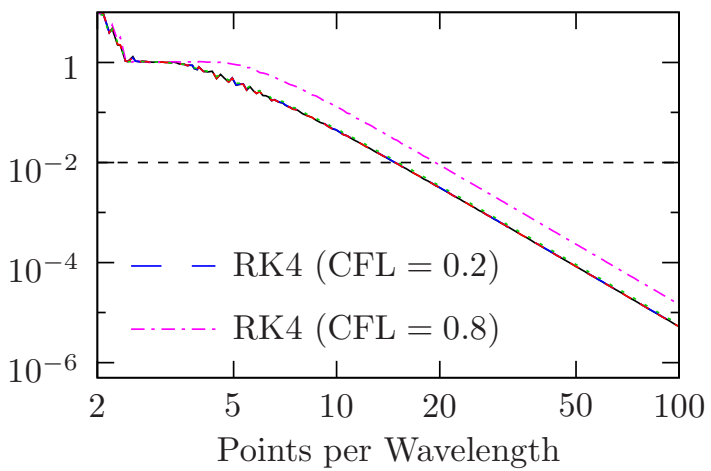

Figure 15: Absolute Error against points per wavelength for several time stepping schemes. (a) and (b) use 7-point explicit spatial derivatives and the 7-point 6th-order filter $F_{6}$, while (c) and (d) use 7-point implicit derivatives and the 9-point filter S8.

both spatial derivatives and temporal integration into one combined scheme, to the author's knowledge no attempt has been made to jointly optimized combined spatial and temporal schemes. (For example, Tam and Webb [3] present both spatial and temporal optimized schemes but optimize each individually rather than jointly optimizing their combination.)

\section{Conclusion}

We have reconsidered the rather classical theory 10] of the harmonic behaviour of finite difference schemes (as shown in figure 1), where a wave with actual wavenumber $\alpha$ gives a numerical derivative with an numerical modified wavenumber $\bar{\alpha}$. Here, we no longer restrict $\alpha$ to be real (though it is emphasized that all finite difference schemes are still applied to real data, even when $\alpha$ is complex). Non-real $\alpha$ corresponds to exponentially growing or decaying waves, which it is argued are ubiquitous in aeroacoustics. Many finite difference schemes have been considered, including the 7-point (6th order) and 15-point (14th order) maximal order schemes, the 7-point DRP schemes of Tam and Webb [3] and Tam and Shen [4] (both 4th order), the 15-point (4th order) DRP scheme of Tam 7], the 9-, 11- and 13-point schemes of Bogey and Bailly [14, and the 2nd, 4th, 6th and 8th order 7-point tri-diagonal schemes of Kim and Lee [8]. In general, figures 4 [7 show that finite-difference schemes optimized over real $\alpha$ perform well only when the waves are of nearly constant amplitude, while for non-constant oscillations the maximal-order schemes are generally preferable (irrespective of the parameters $(M, N, L)$ from section 2 used). In all cases considered here, the optimized DRP schemes give worse phase and group accuracy than the maximal-order schemes for the parameters used by Tam et al. [27] to represent acoustic linings in aeroengine intakes. 
Since the accuracy of maximal-order schemes depends on $|\alpha \Delta x|$ but is insensitive to $\arg (\alpha \Delta x)$, at least for small $|\alpha \Delta x|$, a useful equivalent of points per wavelength for non-constant-amplitude waves is suggested here to be the number of Points Per Complex Wavelength, PPCW $=2 \pi /|\alpha \Delta x|$, which reduces to the usual definition for points per (real) wavelength when $\alpha$ is real. Table1 summarizes the PPCW needed to achieve a given accuracy; for example, the 7-point maximal-order scheme requires $6.1 \mathrm{PPCW}$ to achieve a $1 \%$ error, while the 7-point DRP scheme of Tam and Shen [4] requires 7.2 PPCW in general (although only 4.9 PPW if the wave is of constant amplitude). It is proposed here that, unless there is a priori knowledge of the exact complex wavenumbers present in all directions (e.g. axial, radial and azimuthal directions for waves in a cylinder), computational simulations are likely to be more accurate using the classical maximal-order schemes than using optimized DRP schemes.

An example problem is introduced in section 3 to illustrate this theory. This example problem is intended to test both wave propagation with significant damping per wavelength, and undamped wave propagation over many wavelengths (22 wave lengths in this case). The coefficients of this example problem were inspired by the study of Tam et al. 27]; in particular, the amount of damping, the distance over which the damping is applied, and the axial wavelengths are all broadly comparable. This example problem was solved both using high-accuracy "perfect" time integration and filtering in order to test the behaviour of the spatial derivatives in isolation, and using standard best-practice schemes (including the LDDRK56 4th-order optimized RungeKutta time integration of $\mathrm{Hu}$ et al. 31]). For any given width, the scheme to achieve $1 \%$ error for the fewest points per wavelength is seen from figures 9 and 10 to be the maximal-order scheme. For a 1\% target error, the best scheme of any considered was the 15-point maximal order scheme with the LDDRK56 integrator at $\mathrm{CFL}=0.8$, while for a $0.1 \%$ target error the best scheme of any considered was the 7 -point maximal order scheme, also with the LDDRK56 integrator at CFL $=0.8$.

It should be noted that the example problem of section 3 might appear at first sight to require more PPW than the theory of section 2. This is because the error in section 2 is per derivative calculation, while the error in section 3 is the accumulated error over a large number $24 / \Delta t$ of calculations. As an example, for the 7-point maximal-order scheme, table 1 lists 6.1 PPCW as needed for $1 \%$ error during a single derivative calculation, while 14.1 PPW (13.7 PPCW) are needed for the entire calculation to have below 1\% error. For further details of estimating the $\mathrm{PP}(\mathrm{C}) \mathrm{W}$ required for an entire problem rather than for a single derivative calculation, the reader is referred to Zingg [22].

In practice, schemes of large width are often impractical. In part this is because of problems at boundaries or at the edges of overlapping grids. Schemes of large width also lose their advantage when the underlying data is not sufficiently smooth, such as at a sudden boundary change from hard-walled to lined or if a nonsmooth initial condition were used. While neither of these problems were present in the numerical example here owing to the periodic domain used and the smoothness of the damping and initial conditions, the problem of insufficient smoothness can be expected to be present in general. Hence, both for this example problem and for similar problems, such as that considered by Tam et al. [27], the author recommends the 7-point maximal-order scheme in combination with a 7 -point maximal-order filter $\left(F_{6}\right)$ and the LDDRK56 optimized time integration at a CFL number of 0.8 , with around 15 points per (real) wavelength, or around 14 PPCW, provided a $1 \%$ error is acceptable.

While the analysis presented here has only considered one-dimensional derivatives, these derivatives are often used in practice in $2 \mathrm{D}$ and $3 \mathrm{D}$ simulations, and in such cases the results presented here comparing maximal-order and optimized schemes of the same width will continue to hold; although the optimal stencil width may vary with the number of dimensions as discussed at the end of section 3.3. One possible future avenue of research would be to consider multi-dimensional gradient schemes that are not composed of several one-dimensional derivatives (for example by calculating the derivative at $x_{i, j}$ using function evaluations at diagonal locations such as $\left.x_{i+1, j+1}\right)$. Although such gradient schemes have not been considered here, similar conclusions could be expected in that schemes optimized for only real vectors $\alpha$ would not be expected to perform well for complex $\alpha$.

It is of course unsurprising that schemes optimized only over real $\alpha$ should perform poorly for non-real $\alpha$. Another possible avenue of future research would be to consider optimizing schemes over a range of complex 
$\alpha$, for example by modifying (3) and minimizing instead something like

$$
\int_{0}^{\pi / 2} \int_{0}^{\eta}\left(\Delta x \bar{\alpha}\left(r \mathrm{e}^{\mathrm{i} \theta}\right)-r \mathrm{e}^{\mathrm{i} \theta}\right)^{2} r \mathrm{~d} r \mathrm{~d} \theta
$$

that is, modifying (3) to optimize over $|\alpha \Delta x|<\eta$ and $0<\arg (\alpha \Delta x)<\pi / 2$. However, there are many other possible metrics and optimizations possible even for real $\alpha \Delta x$ with no consensus on the best $[3,[5,[6]$, and optimizing over a range of complex $\alpha \Delta x$ introduces yet more possibilities. Based on the work presented here, the author would be unsurprised if maximal-order schemes were in fact the optimal schemes under some suitable metric accounting for complex $\alpha \Delta x$, on account of their accuracy being independent of $\arg (\alpha \Delta x)$ at least for small $|\alpha \Delta x|$.

Other possibilities for include investigating non-constant-amplitude oscillations for temporal rather than spatial derivatives, and considering jointly optimizing combined spatio-temporal schemes which may allow for the synergy between spatial and temporal schemes (reported in section 3.4) to be better exploited. Despite the existence of joint spatio-temporal schemes [e.g. 3, 32, 33], to the author's knowledge a joint optimization of both simultaneously has not yet been performed.

\section{Acknowledgements}

The author is grateful for financial support from the Royal Society through a University Research Fellowship, and Fellowship of Gonville \& Caius College, Cambridge. Comments by Dr C. Várnai on a draft are also gratefully acknowledged, as are useful discussions with Dr G. Gabard and Prof. D.R. Hixon. A preliminary version of the first part of this paper was presented as part of AIAA Paper 2015-2540 at the 21st AIAA/CEAS Aeroacoustics Conference in Dallas, Texas, USA[1].

\section{Appendix A. Spatial Filters}

In order to remove spurious under-resolved numerical artifacts, a symmetric low-pass spatial filter is applied once each time step. The filtered solution $f_{i}$ is given in terms of the unfiltered solution $u_{i}$ by

$$
f_{i}=u_{i}-\min \{S \Delta t, 1\} D_{i}, \quad \quad \quad \quad \text { where } \quad D_{i}=\sum_{j=-w}^{w} a_{|j|} u_{i+j},
$$

and the filter $D$ is defined by its width $2 w+1$ and coefficients $a_{j}$, and is applied with a strength of $S \Delta t$ (capped at 1 to prevent instability) where $\Delta t$ is the time step and $S$ is a parameter. This strength was found to give optimal results for widely varying $\Delta t$ without varying the parameter $S$. The optimal values of the parameter $S$ depend on the filter, and the values of $S$ used here are given in table A.4.

The frequency responses of the filters used here are plotted in figure A.16, and their coefficients are given in table A.5. The $F_{6}$ filter is the unique filter of half-width $w=3$ that has an accuracy of order $n=6$, meaning that $\left|D_{i}\right|=O\left(\Delta x^{n}\right)$ as $\Delta x \rightarrow 0$. Similarly, the $F_{8}$ filter is the unique filter of half-width $w=4$ that has an accuracy of order $n=8$. The $F_{16,4}$ filter is of width $w=9$ and has accuracy of order $n=16$. The remaining degree of freedom in the $F_{16,4}$ filter is used to specify that $f_{j}=O\left((\pi-\alpha \Delta x)^{4}\right)$ as $\alpha \Delta x \rightarrow \pi$ for $u_{j}=\operatorname{Re}\left(A \mathrm{e}^{\mathrm{i} \alpha x_{j}}\right)$, meaning that higher order damping is required near the Nyquist frequency.

\section{References}

[1] E. J. Brambley, DRP schemes perform poorly for decaying or growing oscillations, AIAA Paper 2015-2540, doi: 10.2514/6.2015-2540, 2015.

[2] P. Moin, Fundamentals of Engineering Numerical Analysis, chap. 2.3, Cambridge, second edn., 2010.

[3] C. K. W. Tam, J. C. Webb, Dispersion-Relation-Preserving Finite Difference Schemes for Computational Acoustics, J. Comput. Phys. 107 (1993) 262-281.

[4] C. K. W. Tam, H. Shen, Direct Computation of Nonlinear Acoustic Pulses using High-Order Finite Difference Schemes, AIAA Paper 93-4325, 1993. 


\begin{tabular}{l|c|c} 
Derivative & Filter & Optimal $S$ \\
\hline 7pt MO & $F_{16,4}$ & 100 \\
7pt DRP [3] & $F_{16,4}$ & 150 \\
7pt DRP [4] & $F_{16,4}$ & 100 \\
15pt MO & $F_{16,4}$ & 2 \\
15pt DRP [7] & $F_{16,4}$ & 2 \\
KL2 [8] & $F_{16,4}$ & 100 \\
KL4 [8] & $F_{16,4}$ & 1 \\
KL6 [8] & $F_{16,4}$ & 1 \\
KL8 (MO) & $F_{16,4}$ & 1 \\
FDo9p [14] & $F_{16,4}$ & 8 \\
FDo11p [14] & $F_{16,4}$ & 2 \\
FDo13p [14] & $F_{16,4}$ & 1.5
\end{tabular}

\begin{tabular}{l|c|c} 
Derivative & Filter & Optimal $S$ \\
\hline 7pt MO & $F_{6}$ & 2 \\
7pt DRP [4] & $F_{6}$ & 2 \\
& & \\
KL2 [8] & $F_{8}$ & 1 \\
KL4 [8] & $F_{8}$ & 1 \\
KL6 [8] & $F_{8}$ & 1 \\
KL8 (MO) & $F_{8}$ & 1 \\
FDo9p [14] & SFo9p & 2.4 \\
FDo11p [14] & SFo11p & 2.4 \\
FDo13p [14] & SFo13p & 2.4
\end{tabular}

Table A.4: Filter strengths $S$ used for the results in figures 915

(a) Linear

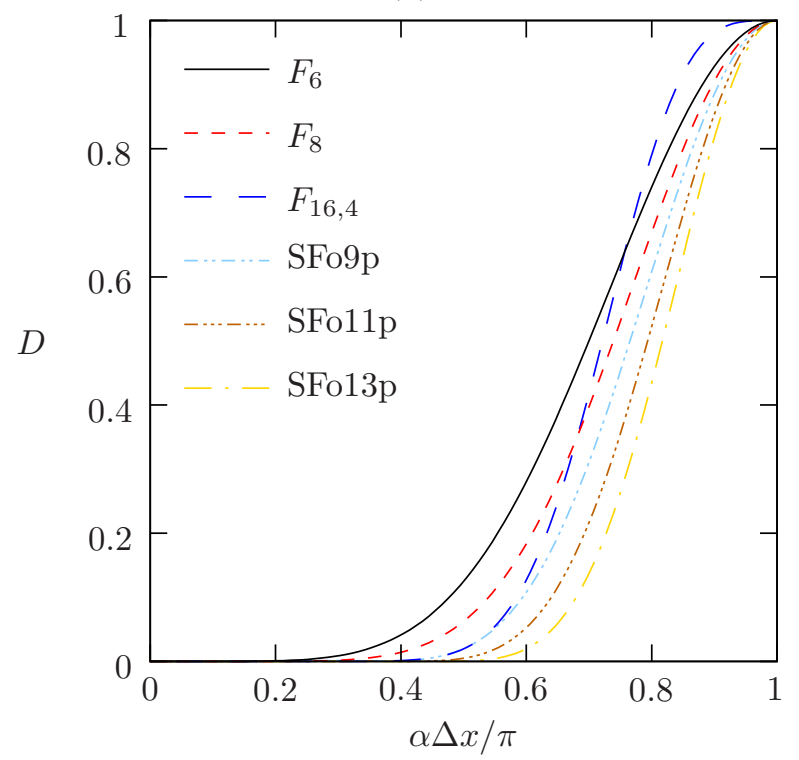

(b) Logarithmic

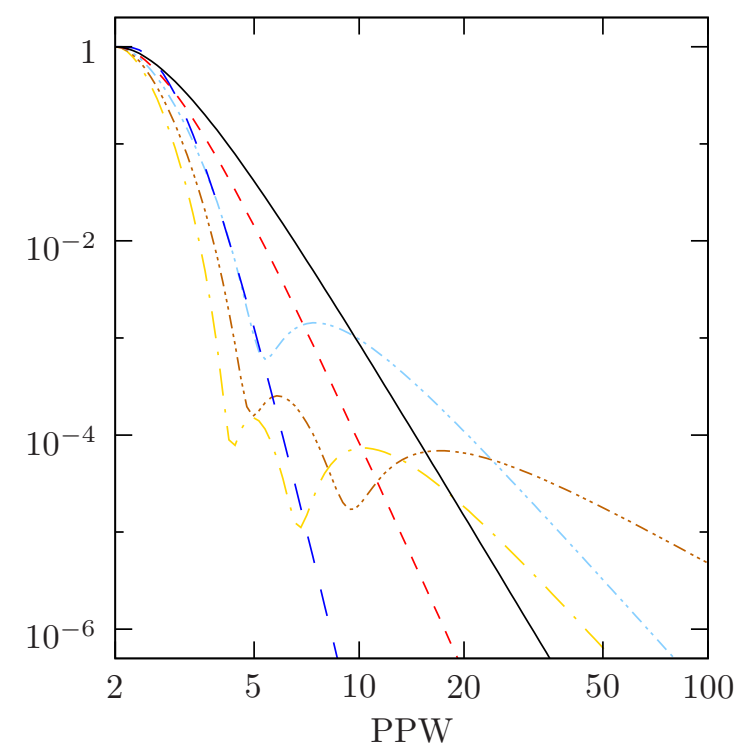

Figure A.16: Frequency response of the symmetric low-pass spatial filters used here.

\begin{tabular}{|c|c|c|c|c|c|c|c|c|c|c|c|c|}
\hline Filter & $n$ & $w$ & $a_{0}$ & $a_{1}$ & $a_{2}$ & $a_{3}$ & $a_{4}$ & $a_{5}$ & $a_{6}$ & $a_{7}$ & $a_{8}$ & $a_{9}$ \\
\hline$F_{6}$ & 6 & 3 & $\frac{5}{16}$ & $-\frac{15}{64}$ & $\frac{3}{32}$ & $-\frac{1}{64}$ & & & & & & \\
\hline$F_{8}$ & 8 & 4 & $\frac{35}{128}$ & $-\frac{7}{32}$ & $\frac{7}{64}$ & $-\frac{1}{32}$ & $\frac{1}{256}$ & & & & & \\
\hline SFo9p [14] & 4 & 4 & & See Ap & endix & 3 of $[14$ & & & & & & \\
\hline SFo11p [14] & 2 & 5 & & See & apper & ix $B$ of & & & & & & \\
\hline SFo13p [14] & 4 & 6 & & & see $A$ & gendix & of [14] & & & & & \\
\hline$F_{16,4}$ & 16 & 9 & $\frac{9295}{32768}$ & $-\frac{3861}{16384}$ & $\frac{1053}{8192}$ & $-\frac{273}{8192}$ & $-\frac{189}{16384}$ & $\frac{135}{8192}$ & $-\frac{69}{8192}$ & $\frac{81}{32768}$ & $-\frac{27}{65536}$ & $\frac{1}{32768}$ \\
\hline
\end{tabular}

Table A.5: Details of symmetric low-pass filters used here. The effect of each filter is $O\left(\Delta x^{n}\right)$ as $\Delta x \rightarrow 0$. 
[5] O. Holberg, Computational Aspects of the Choice of Operator and Sampling Interval for Numerical Differentiation in Large-Scale Simulation of Wave Phenomena, Geophysical Prospecting 35 (1987) 629-655, doi: 10.1111/j.1365-2478.1987.tb00841.x.

[6] J.-H. Zhang, Z.-X. Yao, Optimized Explicit Finite-Difference Schemes for Spatial Derivatives using Maximum Norm, J. Comput. Phys. 250 (2013) 511-526, doi:10.1016/j.jcp.2013.04.029

[7] C. K. W. Tam, Computational Aeroacoustics, chap. 2, Cambridge, 2012.

[8] J. W. Kim, D. J. Lee, Optimized Compact Finite Difference Schemes with Maximum Resolution, AIAA J. 34 (5) (1996) 887-893, doi:10.2514/3.13164

[9] R. Vichnevetsky, F. De Schutter, A Frequency Analysis of Finite Element Methods for Initial Value Problems, in: R. Vichnevetsky (Ed.), Advances in Computer Methods for Partial Differential Equations, AICA, Rutgers Univ., New Brunswick, NJ, 1975.

[10] R. Vichnevetsky, J. B. Bowles, Fourier Analysis of Numerical Approximations of Hyperbolic Equations, SIAM, Philadelphia, 1982.

[11] S. K. Lele, Compact Finite Difference Schmes with Spectral-like Resolution, J. Comput. Phys. 103 (1992) 16-42, doi: 10.1016/0021-9991(92)90324-R.

[12] D. W. Zingg, H. Lomax, Finite-Difference Schemes on Regular Triangular Grids, J. Comput. Phys. 108 (1993) 306-313, doi:10.1006/jcph.1993.1184

[13] C. K. W. Tam, personal communication, 2015.

[14] C. Bogey, C. Bailly, A Family of Low Dispersive and Low Dissipative Explicit Schemes for Flow and Noise Computations, J. Comput. Phys. 194 (2004) 194-214, doi:10.1016/j.jcp.2003.09.003

[15] S. Jakobsson, Frequency Optimized Computation Methods, J. Sci. Comput. 26 (2006) 329-362, doi: 10.1007/s10915-005-9071-7.

[16] S. K. M. Chenoweth, J. Soria, A. Ooi, A New Perspective on Spectral Analysis of Numerical Schemes, Int. J. Numer. Meth. Fluids 68 (2012) 467-482, doi:10.1002/fld.2514

[17] Y. Liu, Globally Optimal Finite-Difference Schemes based on Least Squares, Geophysics 78 (4) (2013) T113-T132, doi: 10.1190/GEO2012-0480.1.

[18] C. Bogey, C. Bailly, Turbulence and energy budget in a self-preserving round jet: direct evaluation using large eddy simulation, J. Fluid Mech. 627 (2009) 129-160, doi:10.1017/S0022112009005801

[19] N. de Cacqueray, C. Bogey, C. Bailly, Investigation of a High-Mach-Number Overexpanded Jet Using Large-Eddy Simulation, AIAA J. 49 (10) (2011) 2171-2182, doi:10.2514/1.J050952

[20] T. W.-H. Sheu, R.-K. Lin, Three-dimensional Bifurcations in a Cubic Cavity due to Buoyancy-Driven Natural Convection, Int. J. Heat and Mass Transfer 54 (2011) 447-467, doi:10.1016/j.ijheatmasstransfer.2010.09.024.

[21] P. K. Mishra, A. K. De, M. K. Verma, V. Eswaran, Dynamics of Reorientations and Reversals of Large-Scale Flow in Rayleigh-Bénard Convection, J. Fluid Mech. 668 (2011) 480-499, doi:10.1017/S0022112010004830

[22] D. W. Zingg, Comparison of High-Accuracy Finite-Difference Methods for Linear Wave Propagation, SIAM J. Sci. Comput. 22 (2) (2000) 476-502, doi:10.1137/S1064827599350320

[23] R. J. Astley, Numerical Methods for Noise Propagation in Moving Flows, with Application to Turbofan Engines, Acoust. Sci. \& Tech. 30 (2009) 227-239, doi:10.1250/ast.30.227

[24] S. W. Rienstra, A Classification of Duct Modes based on Surface Waves, Wave Motion 37 (2003) $119-135$.

[25] E. J. Brambley, N. Peake, Classification of Aeroacoustically Relevant Surface Modes in Cylindrical Lined Ducts, Wave Motion 43 (2006) 301-310.

[26] E. J. Brambley, Surface Modes in Sheared Flow using the Modified Myers Boundary Condition, AIAA Paper 2011-2736, 2011.

[27] C. K. W. Tam, H. Ju, E. W. Chien, Scattering of Acoustic Duct Modes by Axial Liner Splices, J. Sound Vib. 310 (2008) 1014-1035.

[28] L. Tang, J. D. Baeder, Uniformly Accurate Finite Difference Schemes for p-Refinement, SIAM J. Sci. Comput. 20 (3) (1999) 1115-1131, doi:10.1137/S1064827596308354

[29] L. N. Trefethen, Group Velocity in Finite Difference Schemes, SIAM Review 24 (2) (1982) 113-136, URL http://www.jstor.org/stable/2029357

[30] W. H. Press, S. A. Teukolsky, W. T. Vetterling, B. P. Flannery, Numerical Recipes in C, chap. 16.2, Cambridge, second edn., 1992.

[31] F. Q. Hu, M. Y. Hussaini, J. L. Manthey, Low-Dissipation and Low-Dispersion Runge-Kutta Schemes for Computational Acoustics, J. Comput. Phys. 124 (1996) 177-191.

[32] S. A. Karabasov, V. M. Goloviznin, Compact Accurately Boundary-Adjusting High-Resolution Technique for Fluid Dynamics, J. Comput. Phys. 228 (2009) 7426-7451, doi:10.1016/j.jcp.2009.06.037

[33] S. A. Karabasov, V. M. Goloviznin, New Efficient High-Resolution Method for Nonlinear Problems in Aeroacoustics, AIAA J. 45 (12) (2007) 2861-2871, doi:10.2514/1.29796 